\title{
Composition fluctuations in a homopolymer-diblock copolymer mixture covering the three-dimensional Ising, isotropic Lifshitz, and Brasovskiĭ classes of critical universality
}

\author{
Vitaliy Pipich, Dietmar Schwahn, ${ }^{\text {a) }}$ and Lutz Willner \\ Forschungszentrum Jülich GmbH, Institut für Festkörperforschung, D-52425 Jülich, Germany
}

(Received 8 June 2005; accepted 14 June 2005; published online 26 September 2005)

\begin{abstract}
The phase behavior of a three-component polymer blend consisting of a critical mixture of polybutadiene and polystyrene (PB/PS) with varying amount of a symmetric PB-PS diblock copolymer was explored with small-angle neutron scattering. Our focus were thermal composition fluctuations which we discuss in terms of mean field, three-dimensional Ising, isotropic Lifshitz, and Brasovskiu classes of critical universality. Particular attention is spent to the observation of a narrow reentrant two-phase regime and double critical point in the Lifshitz critical regime as well as the Lifshitz line. Critical exponents of the isotropic Lifshitz case are proposed in spite of the demonstrated nonexistence of the isotropic Lifshitz critical point. The Ginzburg number (Gi) and Flory-Huggins parameter were determined over the whole diblock concentration range; Gi changes by three orders of magnitude, two orders of magnitude of that change over a 0.03 diblock concentration interval within the isotropic Lifshitz regime. () 2005 American Institute of Physics. [DOI: $10.1063 / 1.1997136]$
\end{abstract}

\section{INTRODUCTION}

Three-component $\{A ; B\}$ polymer mixtures consisting of a symmetric binary $A / B$ polymer blend of critical composition and of a symmetric $A-B$ diblock copolymer ( $A$ and $B$ symbolize the polymer components) allow the exploration of the three-dimensional (3D) Ising, isotropic Lifshitz, and Brasovskil classes of critical universality. While the 3D Ising and Brasovskiı̌ models, respectively, properly describe polymer blends and diblock copolymers, the isotropic Lifshitz universality class becomes valid when at intermediate diblock concentration the ordered state changes from a spatially uniform to a spatially modulated structure. Lifshitz critical behavior is a quite general phenomenon appearing in systems with competing interactions such as in magnetic systems, ${ }^{1,2}$ ferroelectric liquid crystals, ${ }^{3}$ liquid crystals, ${ }^{4}$ polyelectrolytes ${ }^{5}$ random block copolymers, ${ }^{6}$ and oil/water/ surfactant microemulsions. ${ }^{7}$ In $\{A ; B\}$ polymer blends the modulated structure vanishes continuously in $m=3$ dimensions at the Lifshitz point which is the situation of an isotropic Lifshitz case in ordinary $d=3$ dimension. The isotropic Lifshitz critical universality class is of particular interest because a satisfactory theoretical description of its fluctuation behavior is still missing. ${ }^{89}$ The difficulties of theoretical treatment can be understood from the proposed lower and upper critical dimensions $d_{L}=4$ and $d_{U}=8$, respectively. ${ }^{9}$ The lower critical dimension implies that the isotropic Lifshitz critical point does not exist in ordinary dimension as thermal fluctuations destroy the ordered state whereas the large $d_{U}$ implicates a magnified fluctuation-dominated regime. This is

${ }^{a)}$ Electronic mail: d.schwahn@fz-juelich.de the reason that the isotropic Lifshitz universality class is sometimes mentioned of being only of "purely academic" interest. $^{10}$

Thermal composition fluctuations are always present in multicomponent systems which become particularly important in the vicinity of a critical point. Critical anomaly of thermal fluctuations is a well-known universal phenomenon; it describes large scale properties and is characterized by few parameters such as the dimensions of space and order parameter and by the correlation length of thermal fluctuations. ${ }^{11-13}$ Within the disordered phase one can distinguish three regions: (i) The mean-field regime at temperatures far from the critical point where thermal fluctuations are sufficiently weak to be handled as individual fluctuation modes (Gaussian approximation), (ii) the fluctuation-dominated regime close to the critical point, and (iii) the crossover regime in between when fluctuation modes start to show nonlinear effects. ${ }^{14,15}$ Approximate analytical expressions for the crossover functions are known for the susceptibility and correlation length of the 3D Ising model; they will be discussed in Sec. II. The crossover temperature $T_{X}$ between mean-field and fluctuation-dominated regimes is estimated by the Ginzburg criterion and is characterized by the Ginzburg number $\mathrm{Gi}$ which according to $\mathrm{Gi}=\left|T_{X}-T_{C}\right| / T_{C}$ is defined as a reduced crossover temperature of $T_{X}$ ( $T_{C}$ critical temperature). ${ }^{11-13,16}$

The Lifshitz critical point (LCP) represents a multicritical point where the disordered phase and two ordered phases meet. Within mean-field approximation the critical exponents of the susceptibility $S(0)\left(\propto \tau^{-\gamma}\right)$ and of the correlation length $\xi\left(\propto \tau^{-\nu}\right)$ are evaluated as $\gamma=1$ and $\nu=1 / 4$, respectively. ${ }^{17}$ The reduced temperature field of the critical temperature $T_{C}$ is defined as $\tau=\left|T-T_{C}\right| / T$. Those mean-field critical exponents were observed in the polyethylene; poly(ethylenepropylene) $\{\mathrm{PE} ; \mathrm{PEP}\}$ blend consisting of polymers of relatively 


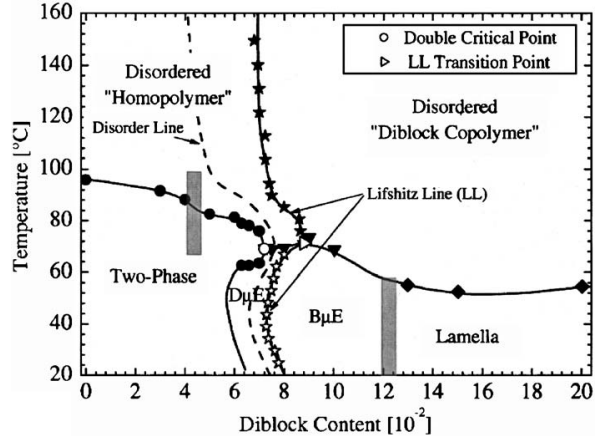

FIG. 1. Temperature-diblock copolymer plane of the $\{\mathrm{dPB} ; \mathrm{PS}\}$ phase diagram. The individual symbols represent the following: The filled circles the Scott line; 3D Ising and isotropic Lifshitz case are separated by a gray box; the open circle the double critical point; the filled and open stars the Lifshitz line at high temperatures of disordered phases and at low temperatures of microemulsion phases, respectively; the filled triangle the disordermicroemulsion transition line; the diamonds the disorder-lamellar ordering transition line; the open triangle the transition point of the two Lifshitz lines; and the second gray box the transition from bicontinuous microemulsion to lamellar phase.

large molar volume ${ }^{17}$ On the other hand a blend mixed with polymers of much smaller molar volume, namely polyethyl ethylene; polydimethylsiloxane $\{\mathrm{PEE}$; PDMS $\}$ shows significantly larger critical exponents which even surpass the corresponding 3D Ising values. ${ }^{18-20}$ Such large critical exponents are reasonable from the already mentioned large upper critical dimension. Another more transparent argument in this direction is related to the amphiphilic effect of copolymers which reduces the interfacial energy and thereby the restoring force of composition fluctuations. The enhanced effect of thermal fluctuations can also be visualized from the Ginzburg number which follows a Gi $\propto 1 / N, \propto 1 / N^{2 / 5}$, and $\propto 1 / N^{1 / 3}$ ( $N$ degree of polymerization) scaling for the ordinary critical point in $A / B$ polymer blends, the LCP in $\{A ; B\}$ mixtures, and the order-disorder transition point in $A-B$ diblock copolymers, respectively. ${ }^{8,21,22}$

Thermal composition fluctuations strongly influence the phase diagram of isotropic Lifshitz systems; the corresponding critical point is suppressed and both ordered phases are separated by a microemulsion channel. ${ }^{17,18,23-27}$ The phase diagram of the present system is depicted in Fig. 1. Depending on the diblock copolymer content one identifies the regimes of 3D Ising, isotropic Lifshitz, and Brasovskiŭ classes of critical universality and the corresponding ordered phases of two domains (spatially uniform), droplet and bicontinuous microemulsion, as well as of lamellar structure (spatially modulated). There exists a borderline of critical points, the so-called Scott line, which is terminated at a double critical point (DCP). Quite generally, the copolymer leads to a better miscibility and to a reentrant two-phase regime with an upper critical solution temperature (UCST) and a lower critical solution temperature (LCST). ${ }^{28,29}$

The focus of the present paper relies on the exploration of thermal composition fluctuations in such a $\{A ; B\}$ polymer blend and their interpretation in terms of the corresponding classes of critical universality. The symbols $A$ and $B$ stand for deuterated polybutadiene ( $\mathrm{dPB}$ ) and polystyrene (PS) chains of molar volumes which were tuned for an ordering
TABLE I. Sample characteristics $\left[{ }^{*} 93 \%(1,4)\right]$.

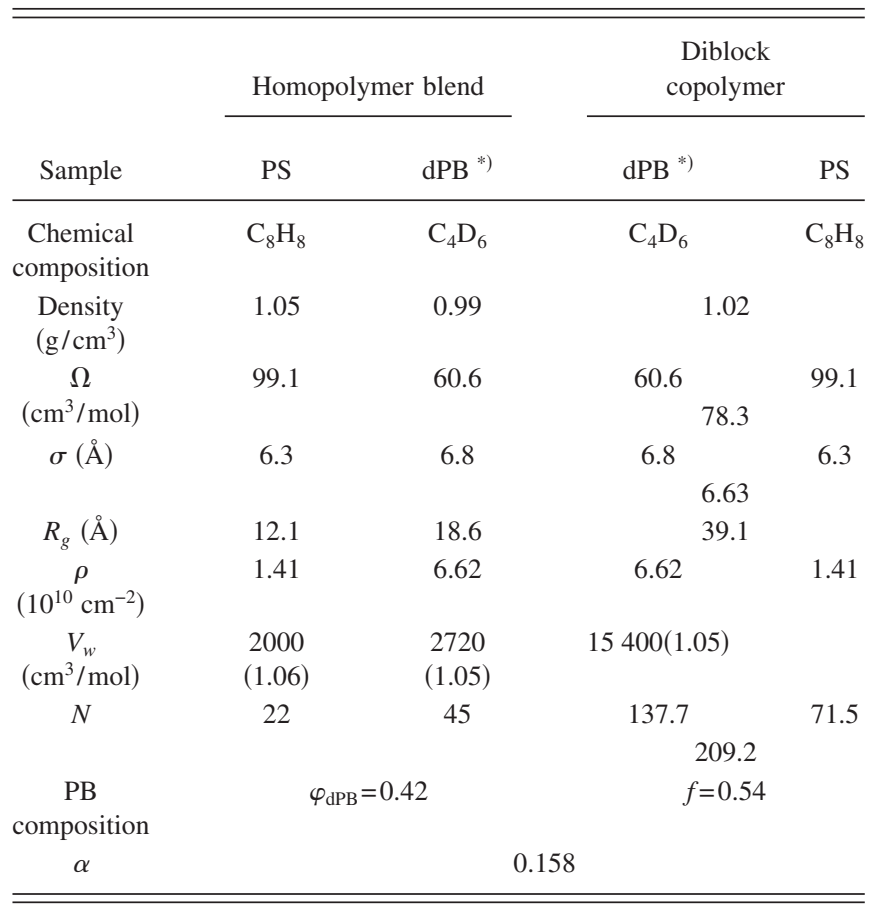

temperature in the range of $100{ }^{\circ} \mathrm{C}$ (Table I). The experimental technique was small-angle neutron scattering (SANS) which is a sensitive tool, in particular, when using deuterated and protonated components for good contrast conditions. The external field parameters were temperature and the amount of the diblock copolymer, the latter one representing a nonordering field. ${ }^{9,10}$ The principal outcome of the present work is a characterization of the isotropic Lifshitz class with respect to their relevant critical exponents and Ginzburg number, as well as a discussion about a nonexistence of the corresponding critical point. The part considering the Ginzburg number has already been published ${ }^{30}$ whereas a forthcoming paper will deal with the ordered phases, in particular, with the microemulsion phase; there we also measured samples with different scattering contrast in order to elucidate the role of the diblock copolymer. ${ }^{31}$

Now we proceed with a summary of the relevant theoretical background, a description of the experimental details before we will represent a detailed analysis and discussion of the scattering results.

\section{THEORETICAL BACKGROUND}

In this section we give a brief description of the underlying theory. After introducing the relevant Hamiltonian we will represent the structure factor $S(Q)$ for binary polymer blends and $\{A ; B\}$ polymer blends in random phase approximation (RPA) and when considering thermal fluctuations.

\section{A. Mean-field theory: Landau-Ginzburg-Wilson Hamiltonian}

The Hamiltonian of a three-component $\{A ; B\}$ polymer blend consisting of a critical binary mixture of $(A / B)$ homopolymers and of the corresponding symmetrical $A-B$ diblock copolymer is given in Eq. (1) 


$$
H=\frac{1}{2} \int d^{3} x\left\{c_{2}(\nabla \Phi)^{2}+c_{4}\left(\nabla^{2} \Phi\right)^{2}+r \Phi^{2}+u \Phi^{4}+u_{6} \Phi^{6}\right\},
$$

and represents a Landau expansion with respect to the order parameter $\Phi(\mathbf{x})$. Within mean-field approximation this Hamiltonian contains the basic thermodynamic features of the present system. ${ }^{9,10,32}$ Thermal composition fluctuations of the component $A$ or $B$ are described by the scalar $(n=1)$ local concentration $\Phi=\Phi(\mathbf{x})\left(\Phi=\Phi_{A}=\left(1-\Phi_{B}\right)\right)$, which is the relevant order parameter and a source of neutron diffraction. The coefficients $c_{2}, c_{4}, r$, and $u$ all depend on the external field parameters temperature and diblock concentration. ${ }^{9}$ Characteristic points of the underlying phase diagram are (i) ordinary critical point when the inverse susceptibility $r=0$ and the other parameters are positive, (ii) ordinary tricritical point when $r=0$ and $u=0$ and the other parameters are positive, (iii) Lifshitz critical point when $r=c_{2}=0$, and (iv) Lifshitz tricritical point, when $r=u=c_{2}=0$ and all the other parameters are positive. The parameters $c_{4}$ and $u_{6}$ have to be positive for stability reasons when $c_{2}$ and $u$ approach zero. Within mean-field theory a tricritical Lifshitz point is predicted for $\{A ; B\}$ blends in the case of equal molar volume of homopolymer and diblock copolymer.

Homopolymer blends are sufficiently well described by the Hamiltonian when $c_{2}>0, c_{4}=0$, and $u_{6}=0$ ( $\Phi$ model $)$. The diblock copolymer's main influence on the Hamiltonian is the reduction of the surface energy as described by the parameter $c_{2}$. This parameter is positive at low copolymer contents, becomes zero at the concentration of the Lifshitz line (LL) and LCP, and negative for larger copolymer content. Equation (1) describes the special case of an isotropic Lifshitz critical point in $d=3$ ordinary dimension as the coefficient of the gradient energy term $(\nabla \Phi)^{2}$ is a scalar which becomes zero in $m=3$ dimensions at the LL, i.e., the ordered modulated phase exists in $m=d=3$ dimensions. The Hamiltonian also accounts for composition fluctuations in the disordered regime as it is related to the structure factor $S(Q)$ via the fluctuation-dissipation theorem. ${ }^{11,32}$ The structure factor is a function of the momentum transfer $Q=(4 \pi / \lambda) \sin (\theta / 2)$ derived from the neutron wavelength $\lambda$ and the scattering angle $\theta$. The expansion of the inverse structure factor $S^{-1}(Q)$ in powers of $Q^{2}$ leads to a good approximation at $Q<1 / \xi$.

$$
\begin{aligned}
S^{-1}(Q) & =S^{-1}(0)+L_{2} Q^{2}+L_{4} Q^{4} \\
& =S^{-1}(0)\left(1+\xi^{2} Q^{2}+\eta^{4} Q^{4} / 4\right) .
\end{aligned}
$$

The coefficients $L_{2}$ and $L_{4}$ are proportional to the $c_{2}$ and $c_{4}$ terms of the Hamiltonian, respectively, and are related to the correlation lengths of the thermal fluctuations according to $\xi=\sqrt{L_{2} / S^{-1}(0)}$ and $\eta=\sqrt[4]{4 L_{4} / S^{-1}(0)}$. The $Q^{4}$ term becomes relevant near the LL, when $L_{2} \rightarrow 0$. In this case the coherence length of thermal fluctuations is described by $\eta$. Another definition is the disorder line (DL) which is derived from the condition of $\xi=\eta$ and describes the situation when one of the correlation functions becomes oscillatory. ${ }^{8}$

The structure factor of binary polymer blends is described by the Ornstein-Zernike equation, which is equivalent to Eq. (2) when $L_{4}=0$ and $\eta=0$. A positive $c_{2}$ is charac- teristic for homopolymer blendlike behavior; the structure factor $S(Q)$ has its maximum at $Q=0$ and the susceptibility is defined according to $r^{-1}=S(0)$. The susceptibility diverges at the critical temperature $T_{C}$ and below $T_{C}$ the blend separates into macroscopic large $A$ and $B$ enriched domains (spatially uniform phase). In the case of a negative $c_{2}$ the structure factor $S(Q)$ shows the characteristic behavior of diblock copolymer melts; the maximum of $S(Q)$ appears at a finite $Q$ $=Q^{*}$ and represents the susceptibility. Within mean-field theory $S\left(Q^{*}\right)$ diverges at the order-disorder critical point $T_{\mathrm{ODT}}$, and forms a lamellar ordered phase (spatially modulated phase) below that temperature. The periodicity is of mesoscopic length scale as determined from $Q^{*}$.

\section{B. Random phase approximation: Polymer blend/diblock copolymer mixture}

Within random phase approximation the structure factor of polymer blend/diblock copolymer mixtures is described according to ${ }^{22,33}$

$$
S^{-1}(Q)=F(Q) / V-2 \Gamma,
$$

with the inverse form factor $F(Q)$ and the Flory-Huggins (FH) parameter $\Gamma=\Gamma_{h} / T-\Gamma_{\sigma}$, where $\Gamma_{h}$ and $\Gamma_{\sigma}$ are entalphic and entropic terms. $F(Q)$ is determined by the partial structure factors $S_{A A}, S_{B B}$, and $S_{A B}$ describing correlations between the corresponding monomers ${ }^{34}$

$$
F(Q) / V=\frac{S_{A A}(Q)+S_{B B}(Q)+2 S_{A B}(Q)}{S_{A A}(Q) S_{B B}(Q)-S_{A B}^{2}(Q)} .
$$

For the critical mixture of $A$ and $B$ homopolymers of equal molar volume $\left(V_{A}=V_{B}\right)$, i.e., $S_{A A}(Q)=S_{B B}(Q)$ and $\Phi_{A}=\Phi_{B}$ $=0.5$, and a symmetric $A B$ diblock copolymer of molar volume $V, F(Q)$ reduces to $F(Q) / V=2 /\left(S_{A A}(Q)-S_{A B}(Q)\right)$. Assuming that the conformation of the polymers in the mixture remains unperturbed Gaussian chains, $F(Q)$ can be written in terms of the Debye function $g_{D}(f, x)=2[f x+\exp (-f x)$ $-1] / x^{2}$ according to

$$
\begin{aligned}
F(x)= & 4 /\left\{\left(1-\Phi_{\mathrm{DB}}\right) \alpha g_{D}(1, x \alpha)-\Phi_{\mathrm{DB}} g_{D}(1, x)\right. \\
& \left.+4 \Phi_{\mathrm{DB}} g_{D}(0.5, x)\right\},
\end{aligned}
$$

where $x=R_{g}^{2} Q^{2} . R_{g}$ is the radius of gyration of the diblock copolymer and $\alpha$ determines the ratio of the molar volumes of the homopolymers relative to the diblock copolymer according to $\alpha=\sqrt{ } V_{A} V_{B} / V$. In the limits of $\Phi_{\mathrm{DB}}=0$ and $=1 F(x)$ becomes the form factor of a binary homopolymer blend and a diblock copolymer melt, respectively. Furthermore, the minimum of $F(x)$ delivers the Flory-Huggins parameter $\Gamma_{C}$ $=F\left(x^{*}\right) /(2 V)$ at the critical point and the corresponding characteristic wave number $Q=Q^{*}$ which leads to the wellknown relationships $\Gamma_{C}=2 / V_{A}$ and $\Gamma_{C}=10.495 / V$ for blends $\left(\Phi_{\mathrm{DB}}=0\right)$ and diblock copolymer melts $\left(\Phi_{\mathrm{DB}}=1\right)$, respectively. The inverse susceptibility is given as

$$
S^{-1}\left(Q^{*}\right)=2\left(\Gamma_{s}-\Gamma\right),
$$

with $Q^{*}=0$ and $\neq 0$ in blend and diblock copolymer case, respectively. In terms of temperature one gets $S\left(Q^{*}\right)$ $=C_{\mathrm{MF}} \tau^{-1}$ with the reduced temperature $\tau=\left(T-T_{C}\right) / T$ and the mean-field critical amplitude $C_{\mathrm{MF}}=1 / 2\left(\Gamma_{C}+\Gamma_{\sigma}\right)=T_{C}^{\mathrm{MF}} / 2 \Gamma_{h}$. 


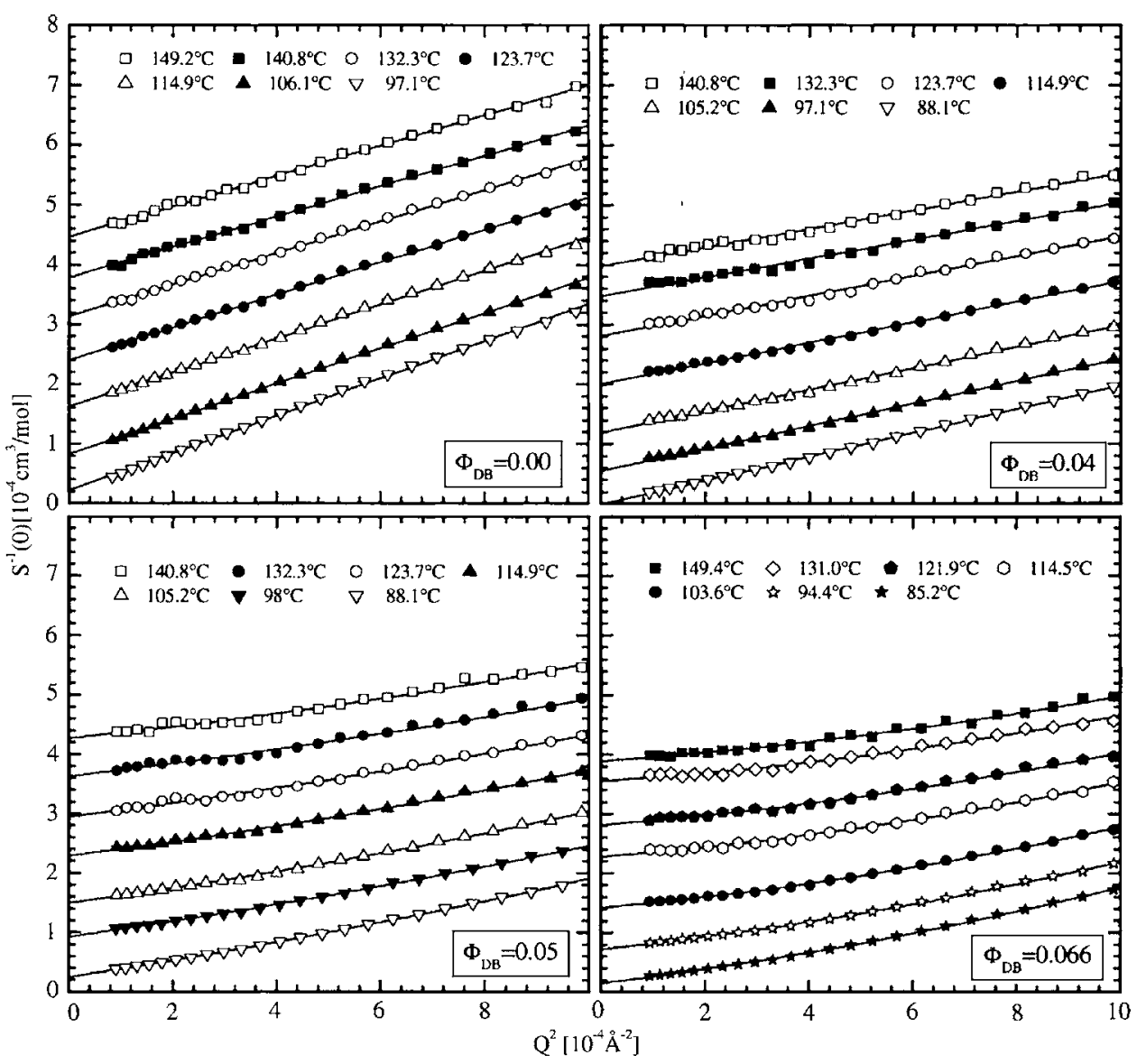

FIG. 2. Structure factor in Zimm representation for four diblock concentrations. Below $\Phi_{\mathrm{DB}}=0.04 S(Q)$ is described by the Ornstein-Zernike law, above 0.04 contributions from the $Q^{4}$ term becomes visible.
The critical exponent $\gamma=1$ and $T_{C}^{\mathrm{MF}}$ are the corresponding mean-field values.

\section{Polymer blend: Mean field to 3D Ising crossover}

Near the critical point mean-field theory becomes invalid. Polymer blends belong to the 3D Ising class of critical universality; close to $T_{C}$ one observes scaling behavior according to $S(Q=0)=C \tau^{-\gamma}$ and $\xi=\xi_{0} \tau^{-\nu}$ with the critical exponents $\gamma=1.239 \pm 0.003$ and $\nu=0.634 \pm 0.001 .^{20}$ This universality class is still the right description when small amounts $\left(\Phi_{\mathrm{DB}}<0.05\right)$ of the PB-PS diblock copolymer were added. Susceptibility $S(0)$ and correlation length $\xi$ were derived from the scattering experiment via the Ornstein-Zernike approximation $S(Q)=S(0) /\left(1+\xi^{2} Q^{2}\right)$ as demonstrated in Fig. 2 for the blend and the $\Phi_{\mathrm{DB}}=0.04$ sample. The crossover of the susceptibility and correlation length from mean field to 3D Ising critical behavior is described in the following as a function of the reduced temperature $\tau$,

$$
\begin{aligned}
& S^{-1}(0)=\left(1 / C_{\mathrm{MF}}\right) \tau Y^{(\gamma-1) / \Delta}\left[1+\frac{u^{*} \nu}{\Delta}\left(\frac{1}{2 \Delta}+\frac{Y}{1-Y}\right)^{-1}\right], \\
& \xi=\xi_{0}^{\mathrm{MF}} \tau^{-1 / 2} Y^{-(2 \nu-1) / 2 \Delta},
\end{aligned}
$$

and the crossover function $Y .{ }^{16}$ The critical exponents $\gamma$ and $\nu$ are those of the 3D Ising model and $\Delta=0.54 \pm 0.03$ is a universal correction-to-scaling exponent. The crossover function $Y$ is either given as an implicit function of the inverse correlation length $\kappa=\xi_{0}^{\mathrm{MF}} / \xi$ or of the reduced temperature $\tau$ according to $(1-Y)=(\bar{u} \Lambda / \kappa) Y^{\nu / \Delta}$ or $(1-Y)^{2} Y^{-1 / \Delta}$ $=\bar{u}^{2} \Lambda^{2} / \tau$, respectively. Equation (7) is valid in the limit of a vanishing microscopic length $(\Lambda \rightarrow \infty)$ which also means that the reduced coupling constant $\bar{u} \rightarrow 0$ because of its definition $\bar{u}=u / u^{*} \Lambda$. The parameters $u$ and $u^{*} \cong 0.472$ represent the coupling constant in the Ginzburg-Landau Hamiltonian in Eq. (1) and the renormalization group (RG) fixed-point coupling constant, respectively. Within the mean-field approach $\bar{u}^{2} \Lambda^{2} / \tau \ll 1$ and $Y=1$ leads to the expected scaling laws $S(0)=C_{\mathrm{MF}} \tau^{-1}$ and $\xi=\xi_{\mathrm{MF}} \tau^{-1 / 2}$ with the corresponding critical amplitudes $C_{\mathrm{MF}}$ and $\xi_{\mathrm{MF}}$. On the other hand one finds within the asymptotic regime of 3D Ising regime $\tau \rightarrow 0$ and $Y \rightarrow 0$ and therefore $Y=\left(\bar{u}^{2} \Lambda^{2} / \tau\right)^{-\Delta}$ leading to the asymptotic scaling laws of the 3D Ising model according to $S(0)=C_{+} \tau^{-\gamma}$ and $\xi=\xi_{0} \tau^{-\nu}$. The ratio of the Ising and mean-field critical amplitudes is derived as $\left(C_{+} / C_{\mathrm{MF}}\right)^{1 /(\gamma-1)}=\bar{u}^{2} \Lambda^{2}$ and $\left(\xi_{0} / \xi_{\mathrm{MF}}\right)^{2 /(2 \nu-1)}=\bar{u}^{2} \Lambda^{2}$. This means that in the case of zero microscopic length $(\Lambda \rightarrow \infty)$ the crossover between the two universality classes is determined by a single parameter, namely, $(\bar{u} \Lambda)^{2}=\left(u / u^{*}\right)^{2}$ which is proportional to the Ginzburg number Gi. In conformity with our earlier work ${ }^{35}$ we define the Ginzburg number as $\mathrm{Gi}=0.069 \bar{u}^{2} \Lambda^{2}$ and finally obtain

$$
\mathrm{Gi}=0.069\left(C_{+} / C_{\mathrm{MF}}\right)^{1 /(\gamma-1)}=0.069\left(\xi_{0} / \xi_{\mathrm{MF}}\right)^{2 /(2 v-1)},
$$

the Ginzburg number represented in terms of the ratio of the Ising and mean-field critical amplitudes and a large exponent $1 /(\gamma-1)[2 /(2 \nu-1)]$ of about 4 as determined from the corresponding Ising critical exponents.

The critical amplitudes of symmetric polymer blends 
fulfilling the Ising model are expressed in terms of the molar volume $V$ according to $C_{+} \propto V^{(2-\gamma)}$ and $\xi_{0} \propto V^{(1-\nu)}$ as derived by Binder and co-worker, ${ }^{12,36}$ while the corresponding amplitudes for the mean-field case are determined from the Flory-Huggins parameter $\Gamma=\Gamma_{h} / T-\Gamma_{\sigma}$ and the "mean-field" critical temperature $T_{C}^{\mathrm{MF}}$, as discussed in context with Eq. (6). The mean-field critical temperature is approximately related to the actual critical temperature $T_{C}$ according to $T_{C}^{\mathrm{MF}}$ $=T_{C} /(1-\mathrm{Gi}){ }^{16,37}$ The last relationship shows that thermal composition fluctuations stabilize the disordered phase by lowering $T_{C}$. These expressions lead to the Ginzburg criterion for polymer blends

$$
\begin{aligned}
& \mathrm{Gi} \propto\left[V^{(2-\gamma)}\left(2 / V+\Gamma_{\sigma}\right)\right]^{1 /(\gamma-1)}, \\
& \mathrm{Gi} \propto\left[V^{(2-2 \nu)}\left(2 / V+\Gamma_{\sigma}\right)\right]^{1 /(2 \nu-1)},
\end{aligned}
$$

which in the case of a zero entropic term $\Gamma_{\sigma}$ becomes the universal scaling law $\mathrm{Gi} \propto 1 / V$. The last expression was originally derived by de Gennes assuming an incompressible polymer melt, i.e., $\Gamma_{\sigma}=0 .{ }^{11,12}$ As already mentioned the Gi represents according to $\mathrm{Gi}=\left|T_{X}-T_{C}\right| / T_{C}$ a reduced temperature of the crossover temperature $T_{X}$.

\section{Effect of thermal fluctuations in the blend/ copolymer mixtures}

Thermal fluctuations are stronger in Lifshitz than in 3D Ising regimes. The origin of this effect is the reduced surface energy expressed by the $c_{2}$ term in the Hamiltonian [Eq. (1)] and with it a reduced threshold force for thermal composition fluctuations. Another expression of this phenomenon is the twice as large upper critical dimension $d_{U}=8$ near the Lifshitz line. ${ }^{8,38}$ An approximate $S(Q)$ for ternary $\{A ; B\}$ blends beyond mean-field approximation was derived by Kielhorn and Muthukumar. ${ }^{21}$ The Hartree approximation within the Brazovskii formalism was applied, which is equivalent to the procedure that was developed by Fredrickson and Helfand for pure diblock copolymer melts. ${ }^{22}$ The structure factor was approximated by a simplified expression according to

$$
S^{-1}(Q)=\frac{a}{b+Q^{2}}+c+d Q^{2}
$$

with the renormalized parameters $a, b, c$, and $d$ considering the effects of thermal fluctuations. ${ }^{21}$ It was assumed that the shape of $S(Q)$ is not affected by thermal fluctuations. The susceptibility $S\left(Q^{*}\right)$ is given as

$$
S^{-1}\left(Q^{*}\right)=2\left[\Gamma_{s} V-\Gamma_{\text {ren }} V\right] / V,
$$

with the renormalized Flory-Huggins parameter $\Gamma_{\text {ren }}$ according to

$$
\Gamma_{\text {ren }} V=\Gamma V-\mathrm{Gi} \cdot F_{X},
$$

which considers the effect of thermal fluctuations via the Ginzburg number Gi and the well-known Flory-Huggins parameter $\Gamma$, whereas $F_{X}$ has to be distinguished for the two cases of the "diblocklike," i.e., $\Phi_{\mathrm{DB}}>\Phi_{\mathrm{LL}}$, and the "blendlike," i.e., $\Phi_{\mathrm{DB}}<\Phi_{\mathrm{LL}}$ behavior. Both expressions for $F_{X}$ are given in the Appendix [Eqs. (A1) and (A2) and in Ref. 21].
The Ginzburg parameter Gi is determined by the degree of polymerization $N$, the diblock copolymer molar volume $V$, and the relative volume fractions of the polymer components $\Phi_{A}, \Phi_{B}$, and $\Phi$ according to $\mathrm{Gi} \propto 1 / \sqrt{\bar{N}}[$ see Eq. (A2)] with the parameter $\sqrt{\bar{N}}=R_{0}^{3} / V$ being the average number of chains in the volume $R_{0}^{3} . R_{0}$ is the end-to-end distance of the polymer $\left(R_{0}=\sqrt{6} R_{g}\right.$ for Gaussian chains). The reciprocal value of $\sqrt{\bar{N}}$ is a measure of the effect of thermal fluctuations. ${ }^{11}$

\section{EXPERIMENT}

The experimental technique applied is SANS which measures thermal composition fluctuations via the structure factor $S(Q)$. The samples are ternary mixtures of two homopolymers PB/PS of critical composition and varying concentrations of the symmetric diblock copolymer PB-PS. One advantage of SANS is the possibility of adjusting the scattering contrast by the exchange of hydrogen and deuterium. We measured the so-called bulk contrast by using $\mathrm{dPB}$ and protonated PS for the homopolymer and diblock copolymer (dPB-PS) in order to explore thermal fluctuations, i.e., local deviations from the average value of the total PS or PB monomer concentration as well as to achieve good scattering contrast conditions.

\section{A. Sample preparation}

The polymers were synthesized by anionic polymerization using similar techniques as already described in Refs. 39 and 40. The PS and the $\mathrm{dPB}$ were synthesized in benzene solution using $s$-butyllithium as initiator. Protonated styrene and perdeuterated butadiene were used as monomers. The diblock copolymer dPB-PS was synthesized by sequential addition of the two monomers. The molecular weight of all polymers and their distribution were determined by size exclusion chromatography in THF relative to PS standards. Transformation to the PB molecular weight was performed by $M_{\mathrm{PB}}=0.581 M_{\mathrm{PS}}^{0.997}$ - derived from the PS and PB MarkHouwink-Sakurada relations in THF. ${ }^{41}$ The higher molecular weight due to deuteration was considered by the ratio of the corresponding monomer molecular weights. The molar volume of the homopolymers is equal to $V_{\mathrm{dPB}}=2720 \mathrm{~cm}^{3} / \mathrm{mol}$, $V_{\mathrm{PS}}=2000 \mathrm{~cm}^{3} / \mathrm{mol}$, and $V_{\mathrm{dPB}-\mathrm{PS}}=15400 \mathrm{~cm}^{3} / \mathrm{mol}$, as shown in Table I. The molar volume of the symmetric diblock copolymer was chosen approximately six times larger, in order to match the ordering temperatures of the homopolymer blend and diblock copolymer. The ratio of the molar volumes of the homopolymers and copolymer is equal to $\alpha=\sqrt{V_{\mathrm{dPB}} V_{\mathrm{PS}}} / V_{\mathrm{dPB}-\mathrm{PS}}=0.15$. The ratio of the two homopolymers was kept constant with the critical value $\left(\varphi_{\mathrm{dPB}}\right.$ $=0.42$ ) as determined from former SANS experiments. The $\mathrm{PS} / \mathrm{dPB}$ blend was prepared by using the freeze-drying method. To achieve a macroscopically homogeneous mixture the homopolymers and diblock copolymer were dissolved in benzene by 2 -h shaking followed by quickly freezing to a temperature of approximately -10 to $-5^{\circ} \mathrm{C}$. Then the solvent was slowly sublimed for $24 \mathrm{~h}$ under high-vacuum conditions. Finally, the cells for the SANS experiment were 
filled in a glovebox under argon atmosphere at a temperature slightly above of the melting temperature. In this way the samples were protected from oxidation.

\section{B. Small-angle neutron scattering}

The small-angle scattering experiments were performed at the diffractometer KWS1 at the FRJ2 reactor of the Forschungszentrum Jülich. ${ }^{42}$ Thermal composition fluctuations were measured in situ at the corresponding temperatures. A two-level temperature heating device was installed inside a vacuum chamber which allowed to keep the temperature of the samples stable within $0.02 \mathrm{~K}$. A PT100 thermocouple determined the temperature of the sample after correction for the gradient between thermocouple and irradiated volume of the sample. The temperature gradient was measured independently under identical conditions with an additional thermocouple at the position of the sample. Before starting the SANS experiments the samples were shaken and annealed at $155^{\circ} \mathrm{C}$ for about $10 \mathrm{~min}$. After changing the temperature we always waited between 10 and 20 min in order to measure the sample in a stable temperature field and under safe equilibrium conditions.

The scattering data were corrected for background, efficiency of the single detector cells, then radially averaged and calibrated in absolute units by a Lupolen secondary standard. The scattered-neutron intensity is given as the macroscopic cross-section $d \Sigma / d \Omega(Q)$ in units of $\mathrm{cm}^{-1}$ which is related to the structure factor $S(Q)$ according to $d \Sigma / d \Omega(Q)$ $=\left(\Delta \rho^{2} / N_{A}\right) S(Q)$ by the contrast factor which is determined from Avogadro's number $N_{A}$ and the difference of the coherent scattering length density $\Delta \rho=\left(\rho_{\mathrm{PS}}-\rho_{\mathrm{dPB}}\right)$ of the PS and dPB polymer components. The scattering length density is evaluated for the monomeric units according to $\rho=\sum c_{i} b_{i} / \Omega$ from the coherent scattering length $b_{i}$ of atom $i$, its fraction $c_{i}$, and the molar volume $\Omega$ of the monomer. The scattering experiments were performed within the momentum-transfer range of $0.002<Q\left(\AA^{-1}\right)<0.1$ with 7 - $\AA$ wavelength of neutrons; 4,8 , and $20 \mathrm{~m}$ were the detector to sample distances and the corresponding collimation distances.

\section{EXPERIMENTAL RESULTS AND INTERPRETATION}

\section{A. Phase diagram}

The temperature-diblock concentration plane of the $\{\mathrm{dPB} ; \mathrm{PS}\}$ phase diagram is depicted in Fig. 1 in the range of $0<\Phi_{\mathrm{DB}}<0.2$. This diagram represents a summary of our experiments. The depicted boundaries are shortly introduced.

\section{Lifshitz line (LL)}

The Lifshitz line divides the phase diagram into two distinct parts. On the left side $\left(\Phi_{\mathrm{DB}}<\Phi_{\mathrm{LL}}\right)$ the system behaves blendlike with the maximum of $S(Q)$ at $Q=0$; one has a disordered phase at high temperatures and two ordered phases at low temperatures, namely, a two-phase regime of macroscopically large domains and a droplet-microemulsion phase. At $\Phi_{\mathrm{DB}}>\Phi_{\mathrm{LL}}$ the mixture behaves diblocklike as the maximum of $S(Q)$ is observed at a finite $Q$ at $Q=Q^{*}$; again one has a disordered phase at high temperatures and two ordered phases at low temperatures, namely, of bicontinuous microemulsion and of lamellar ordered structure. The LL is found at about $\Phi_{\mathrm{DB}}=0.07$ in the high- and low-temperature limits and shifts to larger concentrations near the ordering transitions. The position of the predicted mean-field LL is determined according to $\Phi_{\mathrm{LL}}=2 \alpha^{2} /\left(1+2 \alpha^{2}\right)=0.048$ which is significantly smaller than the experimental high- and lowtemperature values. The bending of the LL near the ordering transitions is caused by thermal composition fluctuations as supported from theoretical considerations. ${ }^{43}$ The LL separates the disordered phase at high temperatures (the so-called "disordered" dLL indicated by the full stars) and the droplet and bicontinuous microemulsion phases at low temperatures (the so-called "microemulsion" $\mu \mathrm{LL}$ indicated by the open stars). Thus, there are two LLs, both meet at the Lifshitz transition point (LTP) as indicated by the open triangle.

\section{Disorder line $(D L)$}

The disorder line is depicted as a dashed line. It was determined from the condition when the two correlation lengths $\xi$ and $\eta$ become equal in size [see discussion in context with Eq. (2)]. On the right side of the DL one of the correlation functions starts to become oscillating. ${ }^{8}$

\section{Disordered "homopolymer" regime}

Near the LL the correlation length $\xi$ approaches zero and $\eta$ becomes dominating overtaking the function of the correlation length; this is the range of isotropic Lifshitz critical behavior. Below $\Phi_{\mathrm{DB}}=0.05 \xi$ represents the correlation length and $\eta$ can be neglected. This is deduced from $S(Q)$ following the Ornstein-Zernike approximation [Eq. (2)] and the underlying thermal fluctuations are described within the three-dimensional Ising model. Crossing the disorder line thermal fluctuations become correlated with the diblock copolymer, i.e., thermal fluctuations start to rearrange the diblock copolymers by accumulating them at their interface.

\section{Scott line}

The Scott line represents a line of critical points which decreases with increasing diblock content and is terminated at the DCP. Within $0.06<\Phi_{\mathrm{DB}}<0.071$ a reentrant two-phase region is found which is limited by a line of upper and lower critical solution temperatures $T_{\mathrm{UCST}}$ and $T_{\mathrm{LCST}}$, respectively. The immiscibility gap $\Delta=T_{\mathrm{UCST}}-T_{\mathrm{LCST}}$ decreases from $15 \mathrm{~K}$ at $\Phi_{\mathrm{DB}}=0.066$ to zero at $\Phi_{\mathrm{DCP}}=0.071$ and $T_{\mathrm{DCP}}=69^{\circ} \mathrm{C}$, which are the coordinates of the double critical point.

\section{Order-disorder line}

The depicted borderline starting from the DCP to larger diblock concentrations represents the order-disorder transition line for the microemulsion and lamellar ordered phases; it covers the droplet and bicontinuous microemulsion phases separated by the LL and a lamellar ordered phase roughly beyond $\Phi_{\mathrm{DB}}=0.12$. 


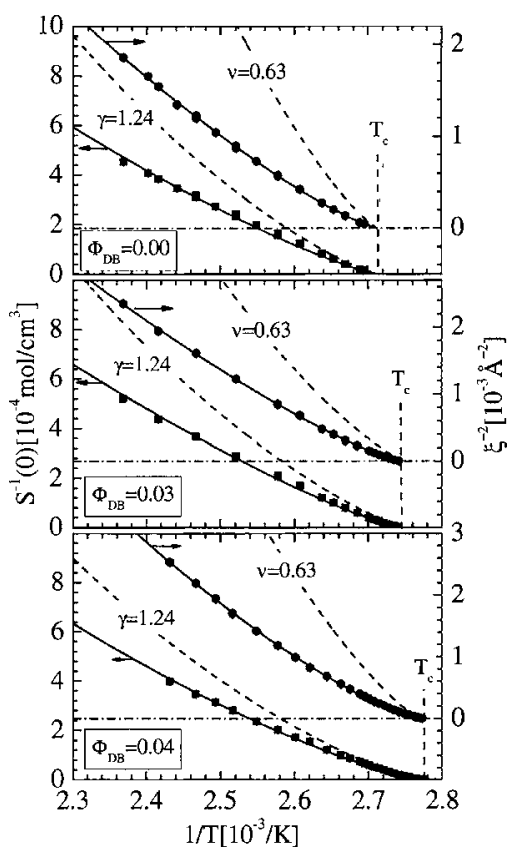

FIG. 3. The inverse susceptibility and square of inverse correlation length vs inverse temperature within the $3 \mathrm{D}$ Ising universality class. The $\Phi_{\mathrm{DB}}=0$, 0.03 , and 0.04 samples follow a crossover function (solid lines) between mean-field (dashed line) and 3D Ising (dotted curve) scaling laws.

\section{B. Structure factor $S(Q)$ below the Lifshitz line}

A selection of $S(Q)$ is depicted in Fig. 2 which was measured for a series of temperatures in the blendlike region between $\Phi_{\mathrm{DB}}=0$ and 0.07 . The structure factors are plotted in Zimm representation, i.e., $S^{-1}(Q)$ vs $Q^{2}$. A strong increase of thermal fluctuations with decreasing temperature is clearly visible from the increase of $S(Q)$. Within the depicted range of $0<Q^{2}\left[\AA^{-2}\right]<10^{-3} S(Q)$ is not affected by the internal polymer structure because the radii of gyration of the polybutadiene homopolymer $\left(R_{g}=18.6 \AA\right)$ and polybutadiene block $(32 \AA)$ fulfill the condition $Q R_{g} \leqslant 1$. Up to $\Phi_{\mathrm{DB}}$ $=0.04$ the scattering is well described by the OrnsteinZernike approximation with $L_{4} \cong 0$. From these experiments the susceptibility $S^{-1}(Q=0)$ and the correlation length $\xi$ were derived. At higher diblock concentrations in the range of $0.05<\Phi_{\mathrm{DB}}<0.075 S(Q)$ shows a weak curvature demonstrating an influencing effect of the $Q^{4}$ term in Eq. (2). The susceptibility $S^{-1}(0)$ and the two correlation lengths $\xi$ and $\eta$ were derived from those measurements.

\section{Susceptibility and correlation lengths in 3D Ising regime}

The inverse susceptibility $S^{-1}(0)$ and the square of the inverse correlation length $\xi^{-2}$ of the three explored samples within the 3D Ising regime at $\Phi_{\mathrm{DB}}=0,0.03$, and 0.04 are depicted versus inverse temperature $1 / T$ in Fig. 3 . The crossover model of Eq. (7) was fitted to $S(0)$ and $\xi$, as shown by the solid lines. The dotted lines correspond to the scaling laws of the asymptotic 3D Ising critical behavior. The crossover model well describes the experimental data and indicates the range of the 3D Ising critical behavior. The $\mathrm{dPB} / \mathrm{PS}$ blend with volume fraction $\varphi_{\mathrm{PB}}=0.42$ is slightly off-critical composition because the minima of $S^{-1}(0)$ and $\xi^{-2}$ do not approach zero; $S^{-1}(0)$ and $\xi^{-2}$ increase again before approaching the critical or spinodal temperature (these points are not shown). The minima identify the binodal whereas the spinodal temperature is determined from the zero extrapolated $S^{-1}(0)$ and $\xi^{-2}$ delivering a gap of $1.7 \mathrm{~K}$ between binodal and spinodal. ${ }^{13}$ Such a metastable gap is not found for the other samples; the minima of $S^{-1}(0)$ and $\xi^{-2}$ of the $\Phi_{\mathrm{DB}}=0.03$ and 0.04 samples are located very near zero either because the diblock copolymer slightly shifts the critical composition or it narrows the metastable regime between spinodal and binodal. Another effect from the addition of diblock copolymer is the increasing relevance of thermal fluctuations which becomes apparent from an enhanced range describing the experimental data by the asymptotic 3D Ising scaling law or what is equivalent to an enlarged Ginzburg number.

The parameters obtained from fitting the crossover function are the critical amplitudes of the two scaling laws $C_{\mathrm{MF}}$, $\xi_{0}$ and $C_{+}, \xi_{+}$, the critical or spinodal temperature $T_{C}\left(T_{S}\right)$ and the Ginzburg number Gi. The corresponding values are summarized in Table II. The Ginzburg number increases by more than a factor of 2 from $\mathrm{Gi}=(0.58 \pm 0.05) \times 10^{-2}$ to $\mathrm{Gi}$ $=(1.5 \pm 0.3) \times 10^{-2}$ when adding $\Phi_{\mathrm{DB}}=0.04$ diblock copolymer to the binary blend. The Ornstein-Zernike law is still the adequate description of $S(Q)$, thus representing a "quasibinary" homopolymer blend.

\section{Susceptibility and correlation lengths in the Lifshitz regime: Scaling approach}

The susceptibility and the two correlation lengths $\xi$ and $\eta$ of four samples covering the regime of the isotropic Lifshitz critical behavior are depicted in Figs. 4(a) and 4(b). Quite generally we observe with increasing $\Phi_{\mathrm{DB}}$ an enlargement of the critical exponents and a decreasing critical temperature. Figure 4(a) shows the inverse susceptibility versus inverse temperature. The solid lines represent a fit of Eqs. (11) and (12) for $Q^{*}=0$ and the corresponding renormalized FH parameter; the strong curvature of the experimental data is well described by this theory. The dashed lines, on the other hand, represent a fit of the scaling law according to

TABLE II. Parameters derived from 3D Ising to mean-field crossover functions of susceptibility $S(0)$ and correlation $\xi[$ Eq. $(7)]\left(\Gamma_{S}\right.$ and $\Gamma_{\sigma}$ in $\left.10^{-4} \mathrm{~mol} / \mathrm{cm}^{3}\right)$; $\Gamma_{h}$ in $\mathrm{mol} \mathrm{K} / \mathrm{cm}^{3}$.

\begin{tabular}{|c|c|c|c|c|c|c|c|c|c|}
\hline$\Phi_{\mathrm{DB}}$ & $\begin{array}{c}T_{C} \\
\left({ }^{\circ} \mathrm{C}\right)\end{array}$ & $\begin{array}{c}\mathrm{Gi} \\
\left(10^{-2}\right)\end{array}$ & $\begin{array}{c}C_{\mathrm{MF}} \\
\left(\mathrm{cm}^{3} / \mathrm{mol}\right)\end{array}$ & $\begin{array}{c}C_{+} \\
\left(\mathrm{cm}^{3} / \mathrm{mol}\right)\end{array}$ & $\xi_{\mathrm{MF}}(\AA)$ & $\xi_{+}(\AA)$ & $\Gamma_{S}$ & $\Gamma_{h}$ & $\Gamma_{\sigma}$ \\
\hline 0.00 & 94.4 & $0.58 \pm 0.05$ & $228 \pm 4$ & 126 & $5.79 \pm 0.02$ & 4.20 & 8.30 & 0.807 & 13.6 \\
\hline 0.03 & 91.6 & $1.05 \pm 0.20$ & $196 \pm 6$ & 125 & $5.10 \pm 0.01$ & 3.99 & 8.47 & 0.933 & 17.0 \\
\hline 0.04 & 87.7 & $1.50 \pm 0.30$ & $220 \pm 2$ & 151 & $4.56 \pm 0.01$ & 3.73 & 8.65 & 0.823 & 14.1 \\
\hline
\end{tabular}




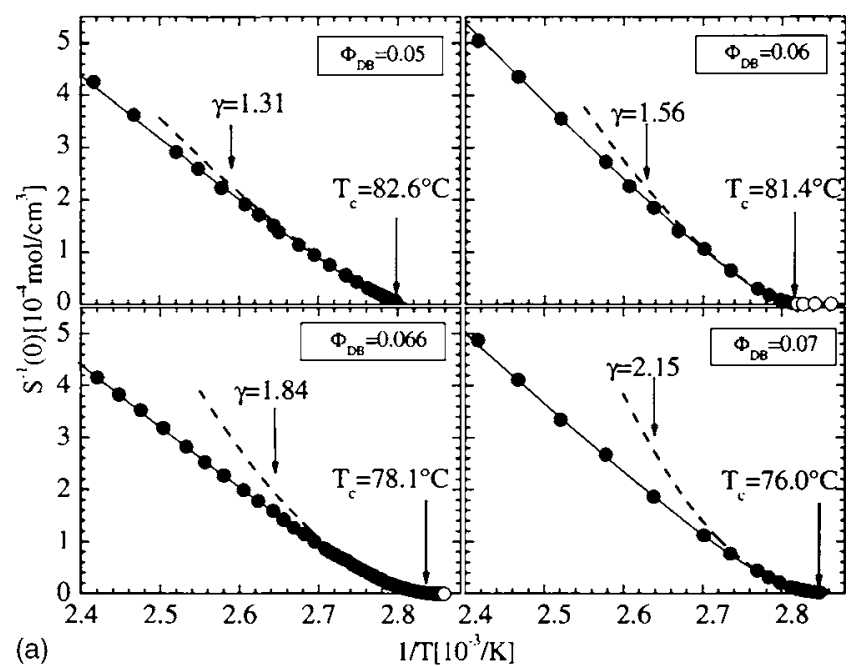

(a)

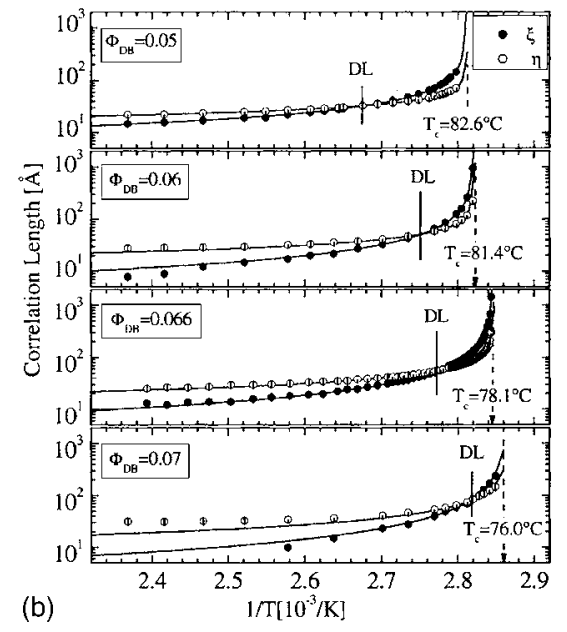

FIG. 4. Inverse susceptibility (a) and the correlation lengths $\xi$ (filled circles) and $\eta$ (open circles) (b) vs inverse temperature within the isotropic Lifshitz universality class. The theory describes the susceptibility (solid lines) and scaling laws [dashed lines in (a) and solid lines in (b)] the parameters near the critical point.

$S^{-1}(0)=C_{+} \tau^{-\gamma}$ describing the data asymptotically close to the critical point. The corresponding correlation lengths $\xi$ and $\eta$ are plotted in Fig. 4(b). Here, the solid lines represent the fit of the two scaling laws $\xi=\xi_{+} \tau^{\nu_{\xi}}$ and $\eta=\eta_{+} \tau^{\nu} \eta$ describing the experiment particularly well between disorder line $(\xi=\eta)$

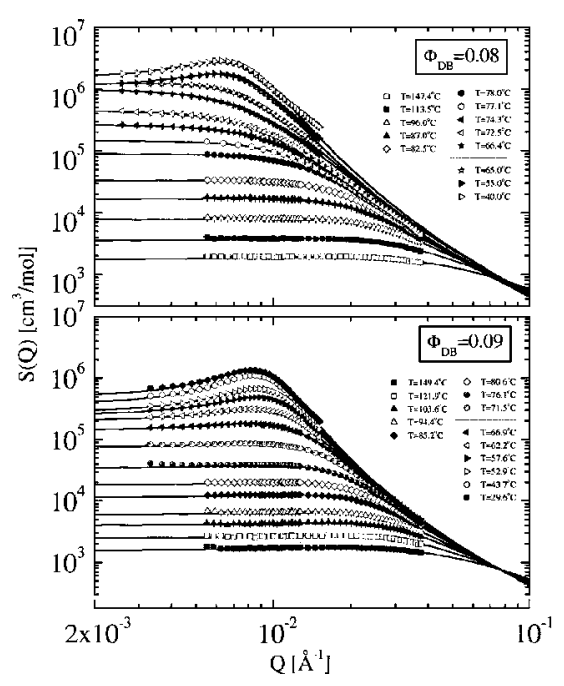

FIG. 5. The scattering profiles within microemulsion channel.

and critical point. The deviations at high temperatures identify the crossover to mean-field behavior. A remarkable increase of the critical exponents is obtained when approaching the Lifshitz line (Table III).

\section{E. Structure factor and susceptibility in the disordered regime above the microemulsion channel}

Figure 5 shows $S(Q)$ from two samples between 0.075 $\leqslant \Phi_{\mathrm{DB}} \leqslant 0.1$ covering the range of the microemulsion channel. For the $\Phi_{\mathrm{DB}}=0.08$ sample the peak position is found both at zero and at finite $Q$ which means that the LL is crossed twice when changing the temperature, whereas a $Q^{*}>0$ was always observed for the $\Phi_{\mathrm{DB}}=0.09$ sample. The structure factor was fitted with the Kielhorn-Muthukumar formalism of Eqs. (10)-(12), as shown by the depicted solid lines. The fit parameters $a, b, c$, and $d$ in Eq. (10) can be formulated in terms of the susceptibility $S\left(Q^{*}\right)$, the forward scattering $S(0)$, the peak position $Q^{*}$, and the segmental length $\sigma$ of the diblock copolymer chain (Appendix). The

TABLE III. Critical parameters of the isotropic Lifshitz critical behavior determined from the isopleths of diblock concentration path using the modified reduced temperature field $\tau_{\mathrm{DCP}}=\left|\left(T-T_{\mathrm{UCST}}\right)\left(T-T_{\mathrm{LCST}}\right)\right| / T^{2}$ in the case of a double critical point. The parameters of the DCP are printed in bold letters. The bottom line represents data from the isothermal path at $69.5^{\circ} \mathrm{C}$. ( *) Sample was measured with light.

\begin{tabular}{|c|c|c|c|c|c|c|c|c|}
\hline$\Phi_{\mathrm{DB}}$ & $\gamma$ & $\begin{array}{c}C_{+} \\
\left(\mathrm{cm}^{3} / \mathrm{mol}\right)\end{array}$ & $\nu_{\xi}$ & $\xi_{+}(\AA)$ & $\nu_{\eta}$ & $\eta_{+}(\AA)$ & $\begin{array}{c}T_{\mathrm{UCST}} \\
\left({ }^{\circ} \mathrm{C}\right)\end{array}$ & $\begin{array}{c}T_{\mathrm{LCST}} \\
\left({ }^{\circ} \mathrm{C}\right)\end{array}$ \\
\hline 0.05 & $1.31 \pm 0.01$ & $163 \pm 7$ & $0.67 \pm 0.01$ & $4.2 \pm 0.1$ & $0.21 \pm 0.01$ & $8.1 \pm 0.2$ & 82.6 & $\cdots$ \\
\hline 0.06 & $1.40 \pm 0.02$ & $67 \pm 4$ & $0.72 \pm 0.01$ & $4.1 \pm 0.2$ & $0.26 \pm 0.01$ & $7.5 \pm 0.3$ & 81.4 & $\ldots$ \\
\hline $0.063^{*}$ & $\ldots$ & $\ldots$ & $\ldots$ & $\ldots$ & $\ldots$ & $\ldots$ & 79 & 62 \\
\hline 0.066 & $1.52 \pm 0.01$ & $41.1 \pm 0.2$ & $0.77 \pm 0.01$ & $2.1 \pm 0.2$ & $0.33 \pm 0.01$ & $6.7 \pm 0.2$ & 78.1 & 62.6 \\
\hline 0.07 & $1.6 \pm 0.01$ & $14.9 \pm 0.7$ & $0.82 \pm 0.01$ & $1.2 \pm 0.2$ & $0.45 \pm 0.01$ & $4.6 \pm 0.3$ & 76.0 & 63.5 \\
\hline 0.071 & $1.61 \pm 0.05$ & $2.3 \pm 0.2$ & $0.89 \pm 0.03$ & $0.16 \pm 0.06$ & $0.43 \pm 0.01$ & $2.8 \pm 0.2$ & \multicolumn{2}{|c|}{$69.1 \pm 0.1$} \\
\hline 0.072 & $1.62 \pm 0.03$ & $0.6 \pm 0.04$ & $0.9 \pm 0.05$ & $0.12 \pm 0.05$ & $0.44 \pm 0.03$ & $2.5 \pm 0.3$ & \multicolumn{2}{|c|}{$69.2 \pm 0.1$} \\
\hline 0.073 & $1.61 \pm 0.02$ & $0.94 \pm 0.07$ & 0.9 & $0.085 \pm 0.003$ & 0.44 & $2.2 \pm 0.1$ & \multicolumn{2}{|c|}{$69.5 \pm 0.2$} \\
\hline 0.075 & $1.62 \pm 0.04$ & $1.4 \pm 0.1$ & 0.9 & $0.13 \pm 0.02$ & 0.44 & $2.76 \pm 0.03$ & \multicolumn{2}{|c|}{$68.8 \pm 0.1$} \\
\hline$T=69.6^{\circ} \mathrm{C}$ & $1.55 \pm 0.15$ & $(27 \pm 2) \times 10^{3}$ & $0.83 \pm 0.09$ & $21 \pm 2$ & $0.39 \pm 0.04$ & $43 \pm 2$ & \multicolumn{2}{|c|}{$\ldots$} \\
\hline
\end{tabular}




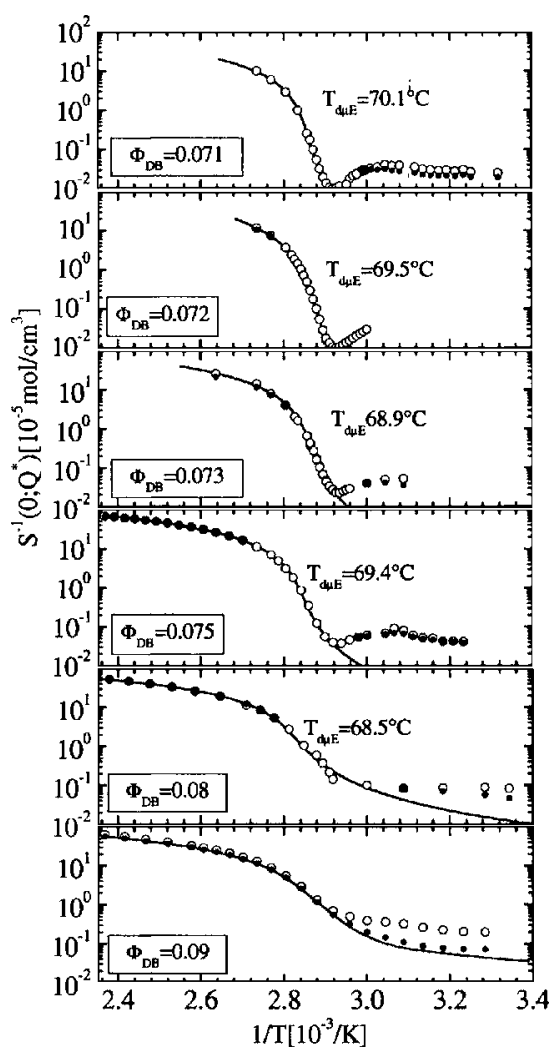

FIG. 6. Near LL inverse susceptibilities $S^{-1}\left(Q^{*}\right)(\odot)$ and $S^{-1}(0)(\odot)$. Within the temperature gap of the Lifshitz lines $S(0)$ represents the susceptibility while at the temperature $T_{d \mu E}$ the disordered phase transforms into a dropletmicroemulsion phase. At this transition line the susceptibility and correlation lengths have their largest values.

corresponding inverse $S\left(Q^{*}\right)$ and $S(0)$ are plotted in Fig. 6, whereas the corresponding correlation lengths $\xi$ and $\eta$ derived from Eq. (2) are not shown here. Within the disordered regime the susceptibility was fitted in terms of the renormalized Flory-Huggins parameter [Eq. (12)] as indicated by the solid lines.

Between $\Phi_{\mathrm{DB}}=0.07$ and 0.09 there exists only a limited temperature interval with $S(0)$ as the susceptibility (open dots) and which is confined by the disordered (dLL) and microemulsion ( $\mu \mathrm{LL}$ ) Lifshitz lines. Above the dLL the homopolymers swell the diblock copolymers with the result of an increasing $Q^{*}$ with increasing diblock copolymer content (more details will be given in a forthcoming paper ${ }^{31}$ ). On the other hand the scattering peak below $\mu \mathrm{LL}$ accounts for the domain-domain correlations of the bicontinuous microemulsion phase.

Another characteristic feature of Fig. 6 is the observed minimum of the susceptibility at around $70{ }^{\circ} \mathrm{C}$. This minimum represents the nearest approach to the DCP at $\Phi_{\mathrm{DB}}$ $=0.071$ and $T=69^{\circ} \mathrm{C}$ (Fig. 1) and is interpreted as representing a borderline between disorder and microemulsion phases. The boundary between disordered and dropletmicroemulsion phases is terminated at the DCP and the LCP at $\Phi_{\mathrm{DB}}=0.088$ and $T=69{ }^{\circ} \mathrm{C}$. The temperature interval of the $Q^{*}=0$ blendlike regime strongly shrinks with increasing diblock content; at $\Phi_{\mathrm{DB}}=0.09$ there is a direct transition from the disordered diblock like to the bicontinuous microemulsion phase, no $Q^{*}=0$ regime becomes visible.

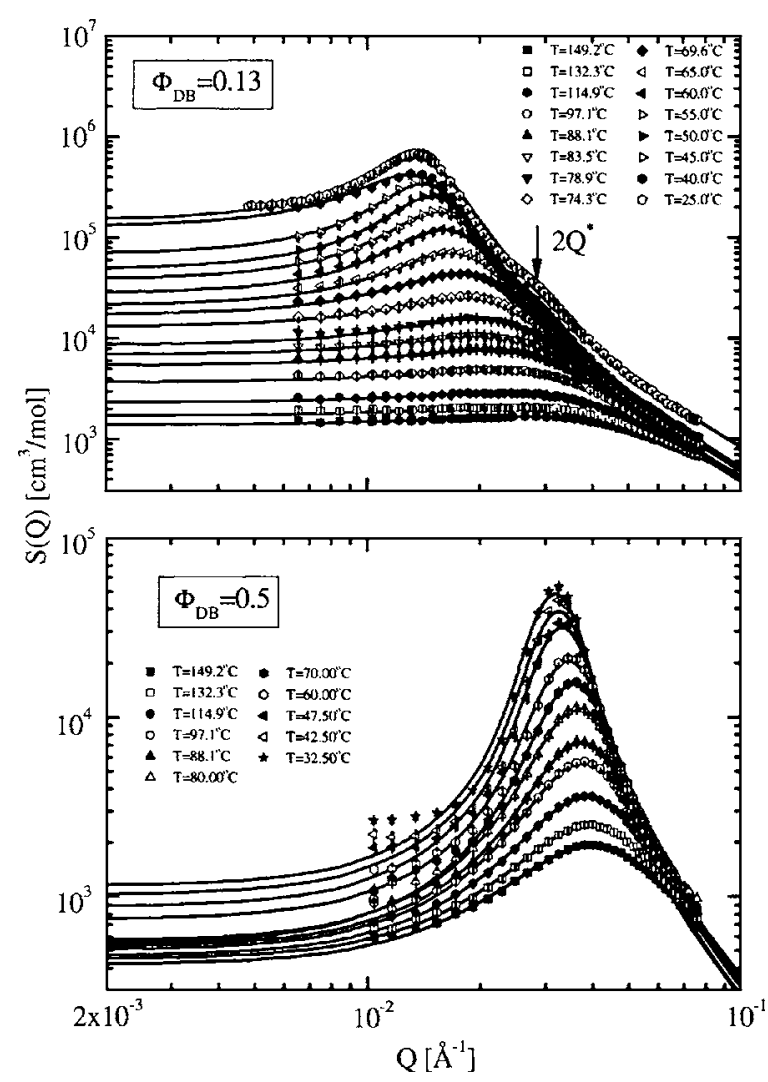

FIG. 7. The scattering function for samples in the Brasovskil regime.

\section{F. $S(Q)$ and susceptibility in Brasovskiĭ universality class}

The structure factor $S(Q)$ of two samples with $\Phi_{\mathrm{DB}}$ $\geqslant 0.13$ is depicted in Fig. 7. This range represents the Brasovskiu critical regime showing an ordering transition to a lamella phase below $T_{\mathrm{ODT}}$ (see phase diagram in Fig. 1). ${ }^{22}$ The structure factor $S(Q)$ shows for all temperatures between 25 and $150{ }^{\circ} \mathrm{C}$ the maximum at finite $Q^{*}$. Here, the procedure of data treatment was as follows: (i) the experimentally obtained structure factor $S(Q)$ was fitted with Eq. (10) taking into consideration the instrumental resolution; the parameters $a, b, c$, and $d$ were extracted. (ii) From these parameters we derived the susceptibility $S\left(Q^{*}\right)$ as depicted in Fig. 8 versus $1 / T$ from which the renormalized $\mathrm{FH}$ parameter $\Gamma_{\text {ren }}$ was determined [Eq. (11)]. From $\Gamma_{\text {ren }}$ we derived the entalphic and entropic terms $\Gamma_{h}$ and $\Gamma_{\sigma}$ of the FH parameter as well as the Ginzburg parameter Gi [Eq. (12)]. The function $F_{X}$ was evaluated from the fit parameters of the $S(Q)$ [Eq. (A1)]. The data are collected in Table IV and the corresponding theoretical $S\left(Q^{*}\right)$ is depicted in Fig. 8 as solid lines showing excellent agreement with the experimental data. The disorderorder transition at $T_{\mathrm{ODT}}$ is indicated by the deviations of the theoretical and experimental susceptibilities and the appearance of a second-order peak of $S(Q)$, as shown in the upper part of Fig. 7. It has to be mentioned that the ordering transition becomes very weak near the LL if compared with pure diblock copolymer melts. ${ }^{44}$

\section{DISCUSSION}

In this section we discuss the relevant parameters derived from $S(Q)$ as they are the critical exponents of the 


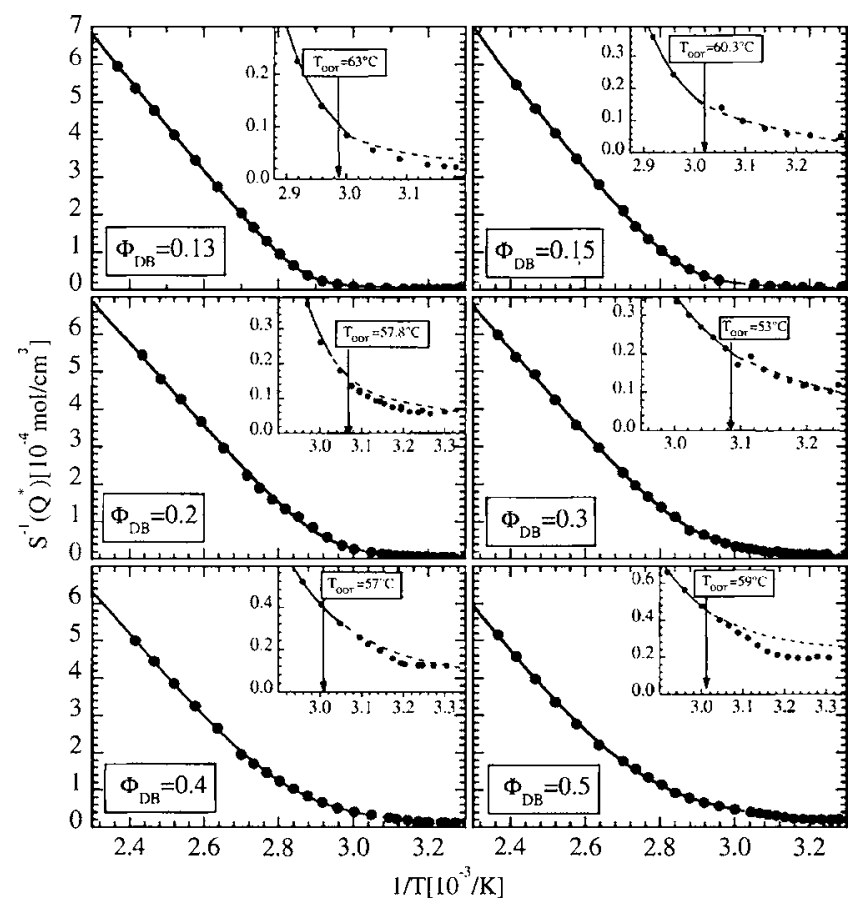

FIG. 8. Susceptibility of $S(Q)$ depicted in Fig. 8 vs inverse temperature. The solid lines represent a fit of theory. The deviations of experiment from fitted curve represent a transition to the ordered lamellar phase.

susceptibility and the correlation lengths, the scaling behavior of $Q^{*}$ near the Lifshitz line, the Ginzburg number, and the Flory-Huggins parameter.

\section{A. Crossover from 3D Ising to isotropic Lifshitz critical regime and double critical point}

The critical exponents of the susceptibility $\gamma$ and of the corresponding correlation lengths $\nu_{\xi}$ and $\nu_{\eta}$ are depicted in Fig. 9 versus the diblock copolymer concentration $\Phi_{\mathrm{DB}}$. These exponents were derived from the corresponding scaling laws valid asymptotically close to the critical points (Scott line). Below $\Phi_{\mathrm{DB}}=0.05$ one finds the critical expo-

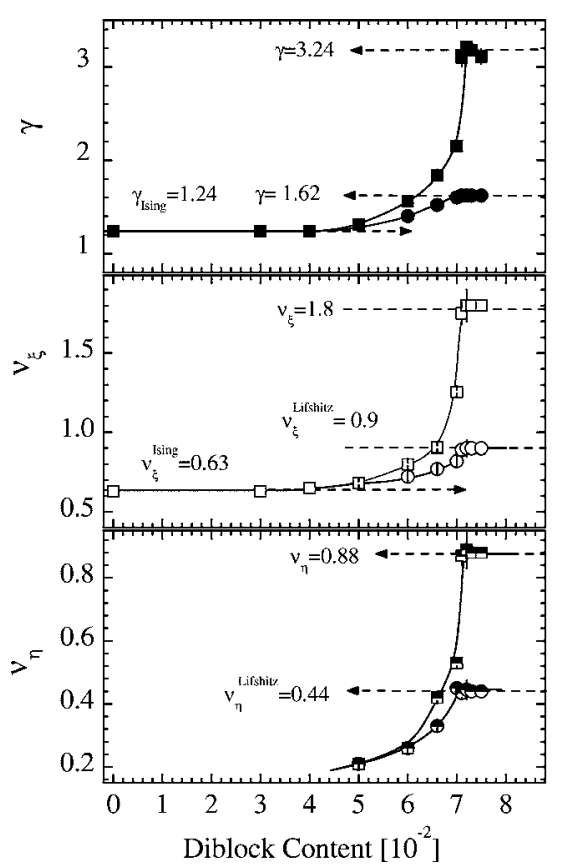

FIG. 9. Crossover of the critical exponents of susceptibility $\gamma$ and correlation lengths $\nu_{\xi}$ and $\nu_{\eta}$. The squares represent the critical exponents derived from a scaling law with the conventional reduced temperature definition. However, as the critical points (Scott line) depend on diblock concentration and are terminated at a double critical point another reduced temperature has to be defined. Within the isotropic Lifshitz regime values of both types of evaluation differ by a factor of 2 .

nents of the 3D Ising model. ${ }^{13,35}$ Between $\Phi_{\mathrm{DB}}=0.05$ and the concentration of the DCP the exponents start to continuously grow to a maximum value of $\gamma=3.24$ and $\nu_{\xi}=1.80$ which then stays constant within the blend like regime. On the other hand, the critical exponent $\nu_{\eta}$ first becomes visible in the Lifshitz critical regime above $\Phi_{\mathrm{DB}}=0.05$ and finally approaches a value of 0.88 (Table III). There are two reasons for the increase of the critical exponents. The first reason is related to the crossover from $3 \mathrm{D}$ Ising to the isotropic Lifshitz class of critical universality which in a former work

TABLE IV. Parameters obtained from Kielhorn-Muthukumar theory for the $\Phi_{\mathrm{DB}}>0.04$ samples. $\left(\Gamma_{S}\right.$ and $\Gamma_{\sigma}$ in $10^{-4} \mathrm{~mol} / \mathrm{cm}^{3} ; \Gamma_{h}$ in $\left.\mathrm{mol} \mathrm{K} / \mathrm{cm}^{3}\right)$.

\begin{tabular}{cccccc}
\hline \hline & $T_{C}, T_{\mu \mathrm{E}}$, & & & \\
$\Phi_{\mathrm{DB}}$ & $T_{\mathrm{ODT}}\left({ }^{\circ} \mathrm{C}\right)$ & $\Gamma_{S}$ & $\Gamma_{h}$ & $\Gamma_{\sigma}$ & $\mathrm{Gi}$ \\
\hline 0.05 & 82.6 & 8.651 & $0.59 \pm 0.01$ & 7.76 & $0.12 \pm 0.01$ \\
0.06 & 81.4 & 8.722 & $0.748 \pm 0.006$ & 12.2 & $0.17 \pm 0.03$ \\
0.066 & 78.1 & 8.755 & $0.62 \pm 0.01$ & 8.60 & $0.40 \pm 0.03$ \\
0.07 & 76.0 & 8.773 & $0.676 \pm 0.007$ & 10.1 & $0.78 \pm 0.10$ \\
0.075 & 68.8 & 8.793 & $0.72 \pm 0.01$ & 11.5 & $0.94 \pm 0.05$ \\
0.08 & 69.5 & 8.810 & $0.63 \pm 0.01$ & 8.90 & $1.10 \pm 0.06$ \\
0.09 & 67.1 & 8.836 & $0.695 \pm 0.01$ & 10.7 & $1.34 \pm 0.05$ \\
0.10 & 66.0 & 8.853 & $0.685 \pm 0.01$ & 10.6 & $1.35 \pm 0.07$ \\
0.13 & 60.3 & 8.869 & $0.70 \pm 0.01$ & 11.0 & $1.40 \pm 0.05$ \\
0.15 & 60.3 & 8.859 & $0.736 \pm 0.007$ & 11.8 & $1.55 \pm 0.11$ \\
0.20 & 57.8 & 8.569 & $0.705 \pm 0.01$ & 11.2 & $1.72 \pm 0.13$ \\
0.30 & 53.0 & 8.306 & $0.70 \pm 0.015$ & 11.1 & $1.87 \pm 0.14$ \\
0.40 & 57.0 & 8.035 & $0.71 \pm 0.03$ & 11.3 & $2.38 \pm 0.22$ \\
0.50 & 59.2 & 6.815 & $0.69 \pm 0.02$ & 10.9 & $3.20 \pm 0.25$ \\
1.00 & 69.0 & 8.791 & $0.68 \pm 0.01$ & 10.0 & $4.00 \pm 0.20$ \\
\hline \hline
\end{tabular}



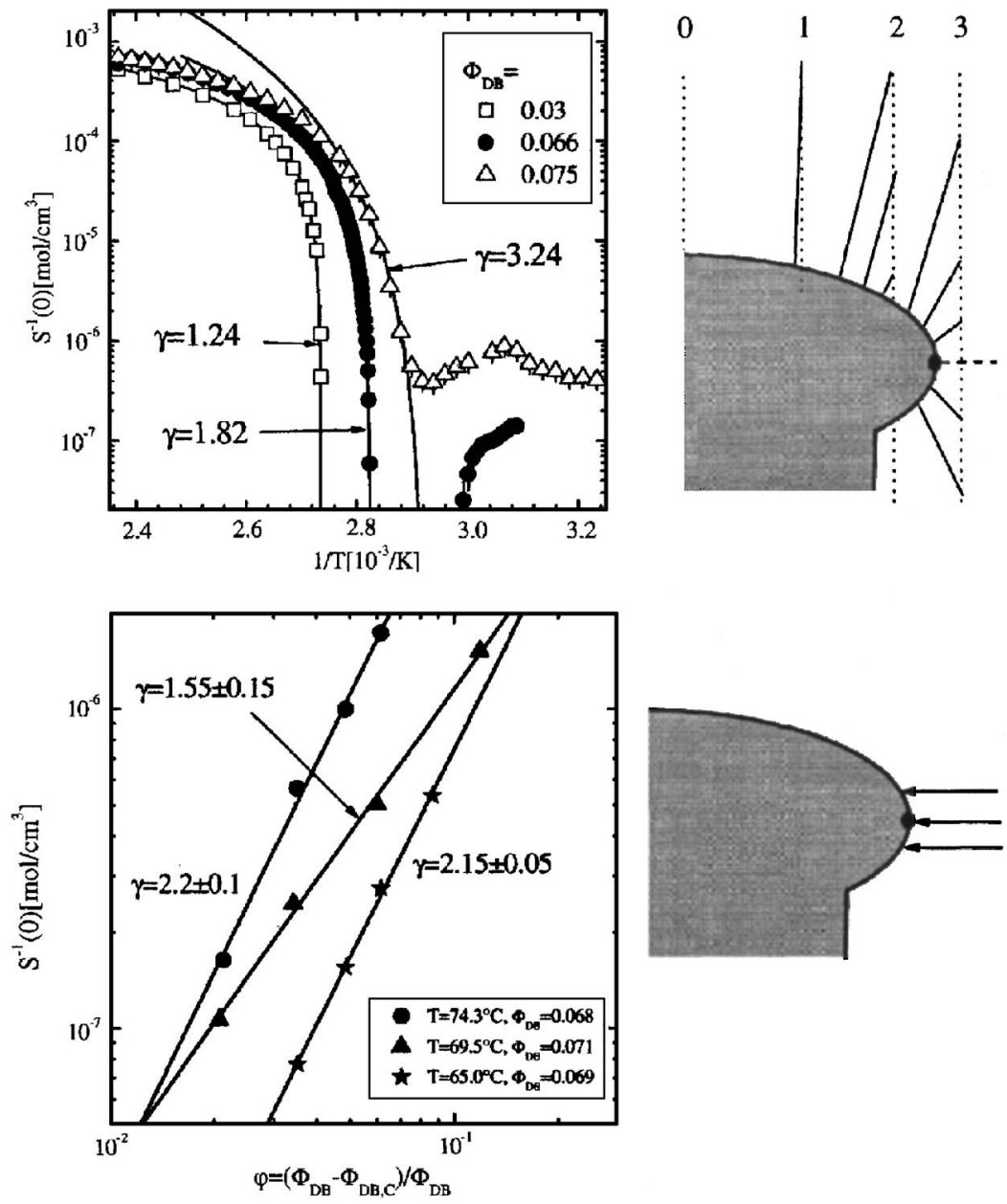

FIG. 10. Experimental approaches towards the double critical point, the isopleths of diblock concentration approach (top), and isothermal (bottom). The isothermal approach to the critical point at $\Phi_{\mathrm{DB}}=0.071$ and $T$ $=69.5^{\circ} \mathrm{C}$ approximately represents a critical path as illustrated in the attached cartoons. was determined as $\gamma=1.62 .^{18}$ The second reason is related to the shape of the Scott line with its DCP (Fig. 1). As illustrated in Fig. 10 the reduced temperature field according to $\tau=\left|T-T_{\mathrm{UCST}}\right| / T$ does not approach the Scott lines perpendicularly; the DCP even represents the extreme case of being approached tangentially (see upper part of Fig. 10). In this case the DCP theory predicts twice as large critical exponents when assuming that the critical line is of approximately quadratic nature at the $\mathrm{DCP} .^{28,29}$ The critical path has to perpendicularly approach the Scott line such as shown in the lower part of Fig. 10; three isotherms are depicted, two of them are slightly above and below the critical temperature of $T_{\mathrm{DCP}}=69{ }^{\circ} \mathrm{C}$ with the reduced diblock copolymer concentration of $\varphi=\left(\Phi_{\mathrm{DB}}-\Phi_{\mathrm{DB}, C}\right) / \Phi_{\mathrm{DB}}$ as the scaling field parameter. The isotherm at $69.5^{\circ} \mathrm{C}$ approximately represents a critical path delivering $\gamma=1.55 \pm 0.15$ and the correlation length exponents (data are not shown) $\nu_{\xi}=0.83 \pm 0.09$ and $\nu_{\eta}$ $=0.39 \pm 0.04$.

Another way to determine the critical exponents from measurements along the isopleths of diblock concentration is based on a redefinition of the reduced temperature field according to $\tau_{\mathrm{DCP}}=\left|\left(T-T_{\mathrm{UCST}}\right)\left(T-T_{\mathrm{LCST}}\right)\right| / T^{2}$ with the upper and lower critical temperatures $T_{\mathrm{UCST}}$ and $T_{\mathrm{LCST}}$, respectively. This approach was suggested by Prafulla et al. ${ }^{29}$ for 3D Ising systems. So, we reexamined the temperature dependence of the susceptibility and correlation lengths by the scaling laws with the newly defined reduced temperature $\tau$ $=\tau_{\text {DCP }}$ and considered the experimental $T_{\text {UCST }}$ and $T_{\text {LCST }}$ depicted in the phase diagram (Fig. 1); the circles in Fig. 9 correspond to those "corrected" critical exponents. Again the critical exponents continuously increase and approach to the values of $\gamma=1.62 \pm 0.03$ and $\nu_{\xi}=0.90 \pm 0.05$ which are consistent with those from the isothermal approach and those derived on a similar polymer mixture. ${ }^{18}$

The graph at the bottom part of Fig. 9 represents the exponent $\nu_{\eta}$. Below $\Phi_{\mathrm{DB}}=0.05$ the exponent $\nu_{\eta}$ is zero due to $\eta=0$, i.e., $L_{4}$ is not measurable whereas for $\Phi_{\mathrm{DB}} \geqslant 0.05$ we observe a continuously increase to $\nu_{\eta}=0.44 \pm 0.03$ when using $\tau=\tau_{\mathrm{DCP}}$ as reduced temperature. The ratio of $\nu_{\xi} / \nu_{\eta}$ $=2.05 \pm 0.16$ is within the error bars consistent with the corresponding ratio of the mean-field value according to $\nu_{\xi} / \nu_{\eta}=0.5 / 0.25=2 .^{8,17}$ The relevant parameters for the samples explored between $\Phi_{\mathrm{DB}}=0.05$ and 0.075 are listed in Table III.

\section{B. Scaling of $Q^{*}$ approaching the Lifshitz line}

The LL is defined as the borderline between blendlike and diblock like behaviors, which is the case when the maximum of $S(Q)$ at a finite $Q=Q^{*}$ for $\Phi_{\mathrm{DB}}>\Phi_{\mathrm{LL}}$ approaches $Q^{*}=0$. An isothermal approach of $Q^{*}$ to the LL is depicted in Fig. 11 for three temperatures; the dependence of $Q^{*}$ is described by a scaling law according to $Q^{*} \propto\left|\Phi_{\mathrm{DB}}-\Phi_{\mathrm{LL}}\right|^{\beta_{Q}}$ which at $48.3{ }^{\circ} \mathrm{C}$ represents a transition from the bicontinu- 


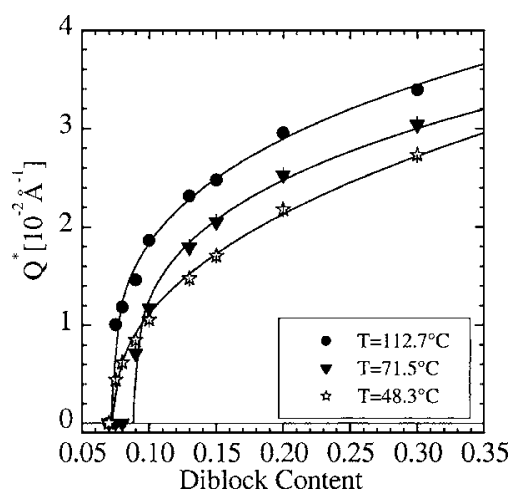

FIG. 11. Scaling behavior of the peak position according to $Q^{*} \propto \mid \Phi_{\mathrm{DB}}$ $-\left.\Phi_{\mathrm{LL}}\right|^{\beta} Q$ within the disordered phase at $112.7^{\circ} \mathrm{C}$, the microemulsion phase at $48.3{ }^{\circ} \mathrm{C}$, and near the disorder-microemulsion transition at $71.5^{\circ} \mathrm{C}$ where the $\mathrm{dLL}$ and $\mu \mathrm{LL}$ lines meet together.

ous to the droplet-microemulsion phase whereas at $71.5^{\circ} \mathrm{C}$ (slightly above the LTP) and at $112.7^{\circ} \mathrm{C}$ a transition from diblocklike to blendlike disordered phase. The critical exponent $\beta_{Q}$ versus temperature is depicted in Fig. 12; a clear distinction of $\beta_{Q}$ is found near $69^{\circ} \mathrm{C}$ separating the highand low-temperature Lifshitz lines. We find the averaged exponents $\left\langle\beta_{Q}\right\rangle=0.42$ and 0.30 for the transition from the bicontinuous to the droplet microemulsion and from diblocklike to blendlike disordered phases, respectively. So, a distinction of two LLs at low and at high temperatures becomes very clear from the critical exponent $\beta_{Q}$. Both LLs meet at the LTP at $\Phi_{\mathrm{DB}}=0.087$ and $T=69^{\circ} \mathrm{C}$. The isopleths of diblock concentration approach to LL (not shown here) from low and high temperatures can be ascribed by a scaling law according to $Q^{*} \propto\left|T-T_{\mathrm{LL}}\right|^{\beta_{Q}}$ with the exponents $\beta_{Q}$ $=0.4$ and 0.3 within the low- and high-temperature phases, respectively, in accordance with those derived from an "isothermal" approach.

Scaling behavior of $Q^{*}$ is predicted from theory in the disordered regime when approaching the Lifshitz critical point but the exponent $\beta_{Q}$ is not known for the isotropic Lifshitz case ${ }^{10}$ whereas in mean-field approximation one gets a $\beta_{Q}=0.5{ }^{9}$

\section{Ginzburg number}

The Ginzburg number is defined as a reduced temperature of the crossover temperature $T_{X}$ between the mean-field

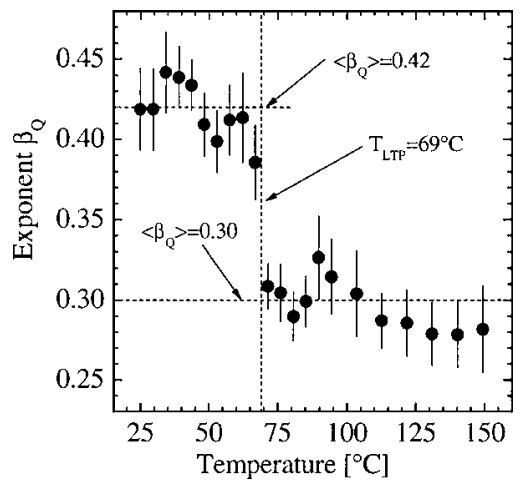

FIG. 12. Temperature dependence of the exponent $\beta_{Q}$. There is a clear transition at $T_{\mathrm{LTP}}$.

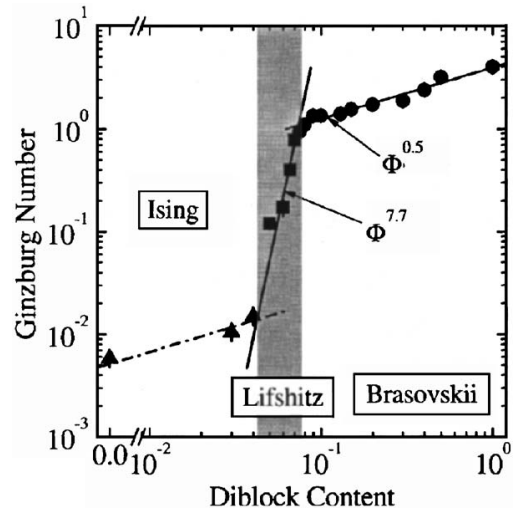

FIG. 13. Ginzburg number from binary blend to diblock copolymer melt.

and the fluctuation-dominated regimes and therefore gives information about the strength of thermal fluctuations. As demonstrated in context with Figs. 3, 4, and 8 the addition of diblock copolymer leads to an enlarged scaling regime and depends on temperature to a stronger curvature of the susceptibility and correlation lengths, i.e., to a larger $\mathrm{Gi}$ and to larger critical exponents, respectively. These observations indicate an increasing role of thermal fluctuations at larger diblock content. All experimental Ginzburg parameters are depicted in Fig. 13, they cover the range from the binary homopolymer blend to the diblockcopolymer melt (see also Table IV). Between the extremes of binary blend and diblock copolymer Gi changes by three orders of magnitude. The strongest change of Gi by two orders of magnitude occurs between $0.04<\Phi_{\mathrm{DB}}<0.08$ within the range of the isotropic Lifshitz critical behavior. Gi can tentatively be described by the power law $\mathrm{Gi} \propto \Phi^{\alpha}$ within the three critical regimes of the 3D Ising, isotropic Lifshitz, and Brasovskiir classes with the exponent $\alpha$ equal to 7.7 and 0.5 for the two latter regimes, respectively.

However, one has to consider that Gi from Ising and the other two regimes was derived from different theories and most probably there exists an unknown factor between them. Within the 3D Ising systems Gi is determined from the ratio of the mean field and 3D Ising critical amplitudes of the susceptibility and correlation length together with the 3D Ising critical exponents $\gamma$ and $\nu$, respectively [see Eq. (8) and Ref. 35]. On the other hand the Gi in the LCP and Brasovskil regimes was identified as $\mathrm{Gi}$ in the second term of the renormalized FH parameter in Eq. (12). Another expression for Gi according to $\mathrm{Gi}=\left(T_{C}^{\mathrm{MF}}-T_{C}\right) / T_{C}$ is obtained from the extrapolation of the crossover function [Eq. (7)] into the classical regime with the crossover temperature identical with the mean-field critical temperature $\left(T_{X}=T_{C}^{\mathrm{MF}}\right)$ [Eqs. (3.6) and (3.7) in Ref. 3]. So, a reevaluation of $\mathrm{Gi}$ from $\mathrm{Gi}=\left(T_{X}^{\mathrm{MF}}\right.$ $\left.-T_{C}\right) / T_{C}$ might be a proper cross-check. In the Ising regime we got the same Gi as it is clear from its definition, while for the LC and Brasovskiŭ regimes we derived an order of magnitude smaller Gi but got a similar dependence with $\Phi_{\mathrm{DB}}$. However, this $\mathrm{Gi}$ is underestimated as the critical temperature can not be determined and $T_{\mathrm{ODT}}$ was chosen instead. So, we can conclude that our experiments give a clear evidence of the very different strength of thermal fluctuations within the 3D Ising, isotropic Lifshitz, and Brasovskil classes of 


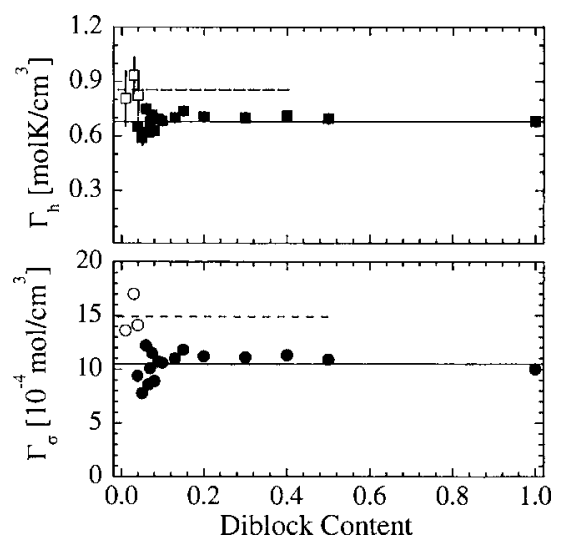

FIG. 14. Enthalpic and entropic terms of the Flory-Huggins parameter vs diblock concentration.

critical universality and, in particular, of a strong change of Gi within the range of the isotropic Lifshitz regime.

\section{Flory-Huggins parameter}

The enthalpic and entropic terms of the FH parameter $\Gamma_{h}$ and $\Gamma_{\sigma}$ from all samples are depicted in Fig. 14 (see also Tables II and IV). The FH parameter of the homopolymer blend $\left(\Phi_{\mathrm{DB}}=0\right)$ and diblock copolymer melt $\left(\Phi_{\mathrm{DB}}=1\right)$ corresponds to the results of Ref. 44. The analysis of the $\Phi_{\mathrm{DB}}<0.05$ samples by the crossover model [Eq. (7)] gives a FH parameter which is close to the homopolymer blend values; their averaged enthalpic and entropic terms are $\left\langle\Gamma_{h}\right\rangle=0.854 \pm 0.05 \mathrm{~mol} \mathrm{~K} / \mathrm{cm}^{3}$ and $\left\langle\Gamma_{\sigma}\right\rangle=(14.9 \pm 1.4)$ $\times 10^{-4} \mathrm{~mol} / \mathrm{cm}^{3}$, respectively (dashed lines in Fig. 14). For the samples fulfilling $\Phi_{\mathrm{DB}}>0.04$ the $\mathrm{FH}$ parameter was calculated from the Kielhorn-Muthukumar theory [Eqs. (11), (12), (A1), and (A2)]; their average values of the entropic and enthalpic terms of $\mathrm{FH}$ parameter are $\left\langle\Gamma_{h}\right\rangle$ $=0.68 \pm 0.05 \mathrm{~mol} \mathrm{~K} / \mathrm{cm}^{3} \quad$ and $\left\langle\Gamma_{\sigma}\right\rangle=(10.5 \pm 1.2)$ $\times 10^{-4} \mathrm{~mol} / \mathrm{cm}^{3}$. Within the error bars these averaged terms of the Flory-Huggins parameter are in agreement with those of the diblock copolymer melt.

In literature the Flory-Huggins theory $\Gamma$ of blends and the corresponding symmetric diblock copolymer is sometimes proposed to be the same when chain-end effects are negligible. However, in more sophisticated theories as the lattice cluster theory the following relationship between the FH parameter of blends and diblock copolymers is proposed:

$$
\Gamma^{\mathrm{DB}}=\left(\Gamma_{h}^{B}-A_{h} / V\right) / T-\left(\Gamma_{\sigma}^{B}-A_{\sigma} / V\right)
$$

which means that the same FH parameter is expected only in the limit of infinite large diblock copolymer chains. ${ }^{45}$ In Ref. 44 the parameters $A_{h}=6775 \mathrm{~K}$ and $A_{\sigma}=19.3$ were found for the polymer components $\mathrm{dPB}$ and PS. For the present system we determined the values $A_{h}=2620 \mathrm{~K}$ and $A_{\sigma}=6.9$ which are nearly three times smaller. An explanation could be an influence of chain-end effects; on the average the chains explored in Ref. 44 were of $15 \%$ smaller molar volume which also became visible by a $31-\mathrm{K}$ lower critical point.

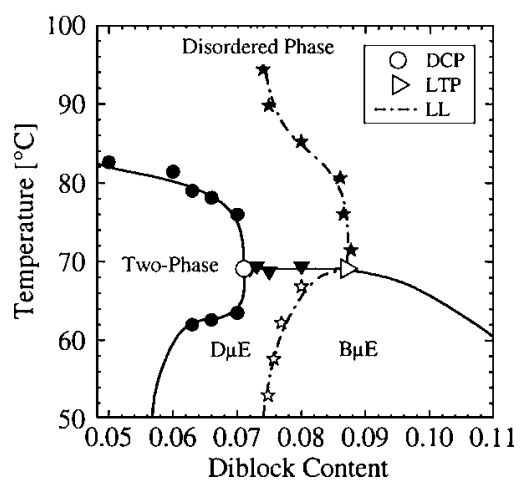

FIG. 15. Phase diagram of the isotropic Lifshitz critical regime near DCP and LTP.

\section{SUMMARY AND CONCLUSION}

The three-component polymer mixture $\{\mathrm{PS} ; \mathrm{dPB}\}$ composed of a PS/dPB critical binary polymer blend and the corresponding symmetric PS-dPB diblock copolymer was explored with small-angle neutron scattering (SANS). The molar volumes of both homopolymers were about the same and about six times smaller than the diblock copolymer $(\alpha=0.158)$. The molar volumes were chosen in order to achieve the ordering transitions within the temperature range between 60 and $100{ }^{\circ} \mathrm{C}$. The external field parameters were temperature and diblock copolymer concentration.

This paper is focused on exploring thermal composition fluctuations within the disordered regime; its characteristic parameters were derived from a detailed analysis of the susceptibility $S\left(Q=Q^{*}\right)$ and the correlation lengths $\xi$ and $\eta$ in terms of disorder-order temperatures, critical exponents, Ginzburg number, and Flory-Huggins parameter. These parameters deliver disorder and Lifshitz lines as well as the phase boundaries of the ordered phases as depicted in the phase diagram (Fig. 1). Four ordered phases were determined, namely, the two-phase regime of macroscopic large domains of enriched PS and APB chains, the droplet- and bicontinuous microemulsion regimes, and the lamellar ordered phase. The droplet-microemulsion phase is observed here for the first time whereas the other ordered phases were also found in similar blends. ${ }^{17,18,23-25}$ Another new observation is the double critical point and, in particular, its observation within the isotropic Lifshitz class of critical universality.

\section{A. Lifshitz line}

The Lifshitz line divides the phase diagram into parts of low and high diblock copolymer concentrations. Actually, there exist two Lifshitz lines, one divides the disordered part into a "blendlike" and "diblocklike" part, whereas the other one the microemulsion phase into a droplet and bicontinuous part. Both Lifshitz lines meet the disorder-microemulsion transition line [Lifshitz transition point (LTP)] at $T=69^{\circ} \mathrm{C}$ and $\Phi_{\mathrm{DB}}=0.087$ (open triangle in Figs. 1 and 15; Fig. 15 shows the Lifshitz critical regime in larger scale) which is $\Delta \Phi_{\mathrm{DB}}=1.5 \%$ far from the DCP. The LTP represents a meeting point of four phases. The observation of DCP and LTP at different positions implies that an isotropic Lifshitz critical 
point does not exist because the conditions of $S^{-1}(0)=0$ and of $L_{2}=\mathrm{c}_{2}=0$ are not fulfilled simultaneously [Eqs. (1) and (2)]. This observation supports our former discussions in context with the proposed lower critical dimension $d_{L}=4$ which does not permit an ordering state in ordinary dimension.

Another observation is the scaling behavior of $Q^{*}$ if approaching the LL from the high diblock concentration side $\left(\Phi_{\mathrm{DB}}>\Phi_{\mathrm{LL}}\right)$; different critical exponents, namely, $\beta_{Q}=0.42$ and 0.3 are found in the disordered and microemulsion regimes, respectively. Apparently they are a sign of distinction (Fig. 12).

\section{B. Thermal composition fluctuations}

Thermal composition fluctuations below the Lifshitz line $\left(\Phi_{\mathrm{DB}}<\Phi_{\mathrm{LL}}\right)$ were analyzed in terms of scaling laws valid in the 3D Ising and isotropic Lifshitz classes. At low diblock concentrations $\Phi_{\mathrm{DB}} \leqslant 0.04$ the blend behaves as 3D Ising system showing the characteristic features of mean field to Ising transition together with a structure factor described by an Ornstein-Zernike law which means that the $L_{2}$ term in Eq. (2) is still the dominating one. But already in this regime the addition of $4 \%$ diblock copolymer leads to an increase of the Ginzburg number from $\mathrm{Gi}=0.58 \times 10^{-2}$ to $1.5 \times 10^{-2}$ and about a $7-\mathrm{K}$ decrease of the critical temperature.

At higher $\Phi_{\mathrm{DB}}>0.04$ a crossover to the isotropic Lifshitz class of critical universality is observed, which is characterized by larger critical exponents and a larger Gi. A larger $\mathrm{Gi}$ implies a larger temperature interval to describe $S(Q=0)$ and both correlation lengths in terms of scaling laws. The crossover from Ising to Lifshitz universality class is accompanied by a change of the $Q$ dependence of $S(Q)$, namely, a reduced $L_{2}$ and an increasingly dominating $L_{4}$ [Eq. (2)]. Both terms correspond to the $c_{2}$ and $c_{4}$ terms in the Hamiltonian [Eq. (1)] and express the surface energy. A positive surface energy stabilizes thermal fluctuations whereas a diminished surface energy near the isotropic Lifshitz critical point leads to enhanced critical fluctuations and an extension of the fluctuation-dominated regime in accordance with the corresponding large upper critical dimension of $d_{U}=8$.

\section{Double critical point (DCP)}

A double critical point is found at $T_{\mathrm{DCP}}=69{ }^{\circ} \mathrm{C}$ and $\Phi_{\mathrm{DCP}}=0.071$ and with it a closed-loop coexistence curve with upper and lower critical solution temperatures in the narrow diblock concentration interval between $0.06>\Phi_{\mathrm{DB}}$ $>0.071$ (Fig. 15). The two-phase gap was detected both by eye and SANS; the blend is transparent below the lower critical temperature and above the upper critical temperature whereas it becomes turbid inside the closed-loop. When entering the two-phase regime from above or from below the extrapolated inverse susceptibility $S^{-1}(0)$ becomes negative and $S(Q)$ shows the characteristic shape found during the early stages of spinodal decomposition. ${ }^{46}$ The gap between the upper and lower critical temperatures becomes zero at the DCP.

In literature reentrant two-phase regions are reported which are surrounded by a disordered phase with no distinc- tion at high and low temperatures. ${ }^{29,47}$ In contrast, the present polymeric blend shows the scenario of a dropletmicroemulsion phase as the "disordered" phase below the reentrant two-phase regime which is separated by a phase boundary (full triangles in Fig. 15) from the disordered phase at high temperatures. The droplet-microemulsion phase must represent a more ordered state if compared with the twophase regime as the corresponding phase separation is driven by entropy. Theoretical descriptions of reentrant two-phase diagrams have to consider at least two distinct modes of interaction as, for instance, van der Waals and hydrogen bonds. Those interactions can be represented by an effective Flory-Huggins free-energy parameter arising from averages over "orientational" degrees of freedom. ${ }^{29}$ For the present polymeric melt the structural differences of homopolymers and diblock copolymers may simulate such distinctive interactions, as the ordering of the diblock copolymers in a droplet microemulsion shows a lower orientational entropy.

\section{Critical exponents near the DCP}

The critical exponents near the DCP depend on the path, this critical point is approached. Only the isothermal path directly delivers the critical exponents as it approaches the DCP perpendicularly. This path is realized when measuring several samples in the disordered state at the critical temperature of $T=69{ }^{\circ} \mathrm{C}$ with varying diblock concentration (see lower part of Fig. 10). On the other hand the isopleths of diblock concentration is the natural path from an experimental point of view. The critical point is passed tangentially at the concentration of the DCP with the result that the critical exponents become twice as large (Fig. 10, top part). In order to determine the critical exponents from this approach one has to redefine the reduced temperature according to $\tau_{\mathrm{DCP}}$ $=\left|\left(T-T_{\mathrm{UCST}}\right)\left(T-T_{\mathrm{LCST}}\right)\right| / T^{2}$ considering both upper and lower critical temperatures. We find the following consistent results. Along the isotherm $\gamma=1.55 \pm 0.15, \nu_{\xi}=0.83 \pm 0.09$, and $\nu_{\eta}=0.39 \pm 0.04$, whereas along the isopleths of diblock concentration $\gamma=1.62 \pm 0.03, \quad \nu_{\xi}=0.90 \pm 0.05, \quad$ and $\nu_{\eta}$ $=0.44 \pm 0.03$.

\section{E. Isotropic Lifshitz critical point (LCP)}

The isotropic Lifshitz critical point is only realized when both DCP and LTP would become identical points. However, after inspection of the phase diagram in Fig. 15 it becomes obvious that there exists no LCP in the $\{\mathrm{dPB} ; \mathrm{PS}\}$ polymer system as DCP and LTP are separated by the $\Delta \Phi_{\mathrm{DB}}=0.015$ interval of diblock concentration. Thus, in spite of the nonexistence of the LCP critical exponents of the susceptibility and correlation lengths can be determined within the ultimate neighborhood of the DCP and LTP. We are confident to attribute these critical exponents to the universality class of the isotropic Lifshitz critical behavior. From a theoretical point of view critical exponents can be evaluated for anisotropic $m$-axial Lifshitz points but not for the isotropic case so far. ${ }^{9}$ Thus, as a result of the present and previous experiments ${ }^{18}$ it seems a realistic conclusion that the isotropic Lifshitz critical behavior in ordinary $d=3$ dimension can be explored within the immediate neighborhood of the expected critical point. 
This also would mean that this class of universality is not anymore only of interest from an "academic point of view.",

\section{F. Brasovskĭ regime}

In the Brasovskil regime above $\Phi_{\mathrm{DB}}=0.13$ the ternary blend shows a first-order disorder-order transition. Experimental indications are higher-order peaks as the secondorder one at $Q=2 Q^{*}$ and a very small but finite step of the susceptibility at the transition temperature $T_{\text {ODT }}$. The corresponding $S(Q)$ are depicted in Fig. 7 and the susceptibility in the disordered regime can be described by the KielhornMuthukumar approach of theory (Fig. 8). ${ }^{21}$

\section{G. Ginzburg number}

The Ginzburg number of the total system as depicted in Fig. 13 was evaluated from the susceptibility by applying theoretical concepts of the 3D Ising crossover function [Eq. (7)] and of LCP and Brasovskiŭ classes of critical universality [Eq. (12)]. Gi in Brasovskiǔ and 3D Ising regimes differs by two to three orders of magnitude and is smoothly interpolated over a $3 \%$ diblock interval in the LCP regime. The center of this crossover is at $6 \%$ diblock content and ends at $7.5 \%$ which is near the DCP. Such a strong change of Gi is understood from a decrease of the surface energy expressed by the coefficient $c_{2}$ in the Hamiltonian [Eq. (1)]. As soon as $c_{2}$ becomes negative (zero at LL), Gi shows a much smaller power-law exponent. An important difference between Ising and Brasovskiı universality is determined by the very different degree of thermal composition fluctuations. ${ }^{30}$

\section{H. Enthalpic and entropic terms}

The enthalpic and entropic terms of the Flory-Huggins parameter are depicted in Fig. 14. Within the Ising universality class $\left(\Phi_{\mathrm{DB}}<0.05\right)$ the enthalpic and entropic terms of the $\mathrm{FH}$ parameter are nearly constant, whereas above $\Phi_{\mathrm{DB}}$ $=0.13$ a slight increase is observed, while within the microemulsion channel both terms sharply decrease by a factor of 2. The different values of the FH parameter of binary polymer blends and the corresponding diblock copolymer as indicated by the dashed and solid lines have already been discussed in Refs. 18 and 44 with the conclusion that the absolute values of the $\mathrm{FH}$ parameters are always smaller in diblock copolymers [Eq. (13)] in accordance with the present work. On the other hand one has to have always in mind that similar to the Ginzburg number the FH parameter is determined from two different theories. Those parameters can be determined with better precision within the 3D Ising model. $^{21,22}$

\section{APPENDIX: RENORMALIZED FH-PARAMETER IN $\{A ; B\}$ BLENDS}

For the blendlike case $\left(\Phi_{\mathrm{DB}}<\Phi_{\mathrm{LL}}\right)$ when the susceptibility is represented according to $S(0)$ and for the diblock case $\left(\Phi_{\mathrm{DB}}>\Phi_{\mathrm{LL}}\right) F_{x}$ in Eq. (12)is determined according to

$$
F_{X}= \begin{cases}R_{g} \sqrt{6 d_{+} b}\left(1-\omega-\omega^{2}\right) / \sqrt{\omega^{2}+2 \omega} & Q^{*}=0 \\ \sqrt{6 x^{*} d_{+}} \frac{b / Q^{* 2}-\sqrt{1+b /\left(d Q^{* 4} S\left(Q^{*}\right)\right)}+2-1 /\left(d Q^{* 2} S\left(Q^{*}\right)\right)}{\sqrt{1 /\left(d Q^{* 2} S\left(Q^{*}\right)\right)-2+2 \sqrt{1+b /\left(d Q^{* 4} S\left(Q^{*}\right)\right)}}} & Q^{*}>0\end{cases}
$$

with the parameter $\omega=1 / \sqrt{d b S(0)}$ and the diblock copolymer radius of gyration $R_{g}$. The parameter Gi is determined by the degree of polymerization $N$, the diblock copolymer molar volume $V$, and the relative volume fractions of the polymer components $\Phi_{A}, \Phi_{B}$, and $\Phi$ according to

$$
\mathrm{Gi}=\frac{N \Gamma_{4}(0,0)}{16 \pi \sqrt{d_{+}^{3}}} \frac{1}{\sqrt{\bar{N}}}
$$

and with the parameters $d$ and $d_{+}$given by $d \equiv d_{+} \sigma^{2} / \Omega$ and $d_{+}=1 /\left\{12\left(\Phi_{A}+f \Phi\right)\left(\Phi_{B}+(1-f) \Phi\right)\right\}$. The parameter $\Gamma_{4}(0,0)$ is the fourth-order vertex function, which was evaluated by the same procedure as used by Leibler ${ }^{33}$ and is a function of $f, \Phi_{A}, \Phi_{B}$, and $\alpha .^{21}$
${ }^{1}$ R. M. Hornreich, M. Luban, and S. Shtrikman, Phys. Lett. 55, 269 (1975).

${ }^{2}$ W. Selke, in Phase Transitions and Critical Phenomena, edited by C. Domb and J. L. Lebowitz (Academic, New York, 1992).

${ }^{3}$ A. Michelson, Phys. Rev. B 16, 577 (1975).

${ }^{4}$ J. H. Chen and T. C. Lubensky, Phys. Rev. A 14, 1202 (1976).

${ }^{5}$ V. Borue and I. Ya. Erukhimovich, Macromolecules 21, 3240 (1988).

${ }^{6}$ G. H. Fredrickson and S. T. Milner, Phys. Rev. Lett. 67, 835 (1991).

${ }^{7}$ G. Gompper and M. Schick, Self-Assembling Amphiphilic System (Academic, London, 1994).

${ }^{8}$ R. Holyst and M. Schick, J. Chem. Phys. 96, 7728 (1992).

${ }^{9}$ H. W. Diehl, Acta Phys. Slov. 52, 271 (2002); H. W. Diehl and M. Shpot, Phys. Rev. B 62, 12338 (2000); M. Shpot and H. W. Diehl, Nucl. Phys. A 612, 340 (2001); M. P. Shpot, Yu M. Pis'mak, and H. W. Diehl, J. Phys.: Condens. Matter 17, 1947 (2005).

${ }^{10}$ R. M. Hornreich and A. D. Bruce, J. Phys. A 11, 595 (1978).

${ }^{11}$ P. G. de Gennes, Scaling Concepts in Polymer Physics (Cornell University Press, Ithaca, 1979).

${ }^{12}$ K. Binder, Adv. Polym. Sci. 112, 181 (1994).

${ }^{13}$ D. Schwahn and K. Mortensen, in Scattering in Polymeric and Colloidal System: Thermal Composition Fluctuations in Polymer Blends Studied with Neutron Small Angle Scattering, edited by W. Brown and K. 
Mortensen (Gordon and Breach, Amsterdam, 2000); D. Schwahn, Adv. Polym. Sci. (to be published).

${ }^{14}$ D. Schwahn, K. Mortensen, and H. Yee-Madeira, Phys. Rev. Lett. 58, 1544 (1987).

${ }^{15}$ F. S. Bates, J. H. Rosedale, P. Stepanek, T. P. Lodge, P. Wiltzius, G. H. Fredrickson, and R. P. Hjelm, Jr., Phys. Rev. Lett. 65, 1893 (1990).

${ }^{16}$ M. A. Anisimov, S. B. Kiselev, J. V. Sengers, and S. Tang, Physica A 188, 487 (1992).

${ }^{17}$ F. S. Bates, W. W. Maurer, T. P. Lodge, M. F. Schulz, M. W. Matsen, K. Almdal, and K. Mortensen, Phys. Rev. Lett. 75, 4429 (1995).

${ }^{18}$ D. Schwahn, K. Mortensen, H. Frielinghaus, K. Almdal, and L. Kielhorn, J. Chem. Phys. 112, 5454 (2000).

${ }^{19}$ D. Schwahn, K. Mortensen, H. Frielinghaus, and K. Almdal, Phys. Rev. Lett. 82, 5056 (1999).

${ }^{20}$ J. C. Le Guillon and J. Zinn-Justin, Phys. Rev. Lett. 39, 95 (1977); J. Zinn-Justin, J. Phys. (Paris) 42, 783 (1981); J. C. Le Guillon and J. Zinn-Justin, ibid. 48, 19 (1987).

${ }^{21}$ L. Kielhorn and M. Muthukumar, J. Chem. Phys. 107, 5588 (1997).

${ }^{22}$ G. H. Fredrickson and E. Helfand, J. Chem. Phys. 87, 697 (1987).

${ }^{23}$ F. S. Bates, W. W. Maurer, P. M. Lipic, M. A. Hillmyer, K. Almdal, K. Mortensen, G. H. Frederickson, and T. P. Lodge, Phys. Rev. Lett. 79, 849 (1997).

${ }^{24}$ M. A. Hillmyer, W. W. Maurer, T. P. Lodge, F. S. Bates, and K. Almdal, J. Chem. Phys. 103, 4814 (1999).

${ }^{25}$ T. L. Morkved, P. Stepanek, K. Krishnan, F. S. Bates, and T. P. Lodge, J. Chem. Phys. 114, 7247 (2001).

${ }^{26}$ V. Pipich, D. Schwahn, and L. Willner, Physica B 350, e897 (2004).

${ }^{27}$ D. Düchs, V. Ganesan, G. H. Fredrickson, and F. Schmid, Macromolecules 36, 9237 (2003); D. Düchs and F. Schmid, J. Chem. Phys. 121, 2798 (2004); M. Müller and F. Schmid, Adv. Polym. Sci. (in press).

${ }^{28}$ R. Johnston, N. Clark, P. Wiltzius, and D. Cannell, Phys. Rev. Lett. 54, 49 (1985).
${ }^{29}$ B. V. Prafulla, T. Narayanan, and A. Kumar, Phys. Rev. A 46, 7456 (1992).

${ }^{30}$ V. Pipich, D. Schwahn, and L. Willner, Phys. Rev. Lett. 94, 117801 (2005).

${ }^{31}$ V. Pipich, D. Schwahn, and L. Willner (unpublished).

${ }^{32}$ D. I. Uzunov, Theory of Critical Phenomena (World Scientific, Singapore, 1993).

${ }^{33}$ L. Leibler, Macromolecules 13, 1602 (1980).

${ }^{34}$ D. Broseta and G. H. Fredrickson, J. Chem. Phys. 93, 2927 (1990).

${ }^{35}$ D. Schwahn, G. Meier, K. Mortensen, and S. Janssen, J. Phys. II 4, 837 (1994).

${ }^{36}$ A. Sariban and K. Binder, J. Chem. Phys. 86, 5859 (1987).

${ }^{37}$ J. V. Sengers, in Supercritical Fluids: Fundamentals for Application: Effect of Critical Fluctuations on Thermodynamic and Transport Properties of Supercritical Fluids, edited by E. Kiran and J. M. H. Levelt Sengers (Kluwer Academic, Dordrecht, 1994).

${ }^{38}$ J. F. Nicoll, G. F. Tuthill, T. S. Chang, and H. E. Stanley, Physica 86-88, 618 (1977).

${ }^{39}$ M. Morton and L. J. Fetters, Rubber Chem. Technol. 48, 359 (1975).

${ }^{40}$ N. Hadjichristidis, H. Iatrou, S. Pispas, and M. Pitsikalis, J. Polym. Sci., Part A: Polym. Chem. 38, 3211 (2000).

${ }^{41}$ L. J. Fetters, N. Hadjichristidis, J. S. Lindner, and J. W. Mays, J. Phys. Chem. Ref. Data 23, 619 (1994).

${ }^{42}$ Description of scattering instruments at FRJ2-Jülich; www.neutronscattering.de

${ }^{43}$ A. Kudlay and S. Stepanow, Macromol. Theory Simul. 11, 16 (2002).

${ }^{44}$ H. Frielinghaus, B. Abbas, D. Schwahn, and L. Willner, Europhys. Lett. 44, 606 (1998)

${ }^{45}$ J. Dudowicz and K. F. Freed, Macromolecules 26, 213 (1993).

${ }^{46}$ D. Schwahn, S. Janßen, and T. Springer, J. Chem. Phys. 97, 8775 (1992).

${ }^{47}$ R. Goldstein and J. Walker, J. Chem. Phys. 78, 1492 (1983); R. Goldstein, ibid. 83, 1246 (1985). 